\title{
Isochronal age-mass discrepancy of young stars: SCExAO/CHARIS integral field spectroscopy of the HIP 79124 triple system
}

\author{
Ruben Asensio-Torres ${ }^{1}$, Thayne Currie ${ }^{2,3,4}$, Markus Janson $^{1}$, Silvano Desidera ${ }^{5}$, Masayuki Kuzuhara ${ }^{6,7}$, \\ Klaus Hodapp ${ }^{8}$, Timothy D. Brandt ${ }^{9}$, Olivier Guyon ${ }^{3,6,10,11}$, Julien Lozi ${ }^{3}$, Tyler Groff ${ }^{12}$, Jeremy Kasdin ${ }^{13}$, \\ Jeffrey Chilcote ${ }^{14}$, Nemanja Jovanovic ${ }^{15}$, Frantz Martinache ${ }^{16}$, Michael Sitko ${ }^{17}$, Eugene Serabyn ${ }^{18}$, Kevin Wagner ${ }^{10}$, \\ Eiji Akiyama ${ }^{19}$, Jungmi Kwon ${ }^{20}$, Taichi Uyama ${ }^{21}$, Yi Yang ${ }^{22}$, Takao Nakagawa ${ }^{20}$, Masahiko Hayashi ${ }^{7}$, \\ Michael McElwain ${ }^{12}$, Tomoyuki Kudo ${ }^{3}$, Thomas Henning ${ }^{23}$, and Motohide Tamura ${ }^{6,7,24}$ \\ (Affiliations can be found after the references)
}

Received 20 November 2018 / Accepted 18 December 2018

\begin{abstract}
We present SCExAO/CHARIS 1.1-2.4 $\mu$ m integral field direct spectroscopy of the young HIP 79124 triple system. HIP 79124 is a member of the Scorpius-Centaurus association, consisting of an A0V primary with two low-mass companions at a projected separation of $<1^{\prime \prime}$. Thanks to the high quality wavefront corrections provided by SCExAO, both companions are decisively detected without the employment of any PSFsubtraction algorithm to eliminate quasi-static noise. The spectrum of the outer C object is very well matched by Upper Scorpius M4 \pm 0.5 standard spectra, with a $T_{\text {eff }}=2945 \pm 100 \mathrm{~K}$ and a mass of $\sim 350 M_{\text {Jup }}$. HIP $79124 \mathrm{~B}$ is detected at a separation of only 180 mas in a highly-

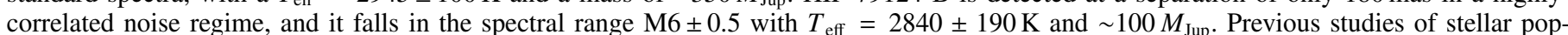
ulations in Sco-Cen have highlighted a discrepancy in isochronal ages between the lower-mass and higher-mass populations. This could be explained either by an age spread in the region, or by conventional isochronal models failing to reproduce the evolution of low-mass stars. The HIP 79124 system should be coeval, and therefore it provides an ideal laboratory to test these scenarios. We place the three components in a colormagnitude diagram and find that the models predict a younger age for the two low-mass companions ( $\sim 3 \mathrm{Myr})$ than for the primary star ( 6 Myr). These results imply that the omission of magnetic effects in conventional isochronal models inhibit them from reproducing early low-mass stellar evolution, which is further supported by the fact that new models that include such effects provide more consistent ages in the HIP 79124 system.
\end{abstract}

Key words. binaries: close - stars: low-mass - stars: pre-main sequence - techniques: imaging spectroscopy - planets and satellites: detection

\section{Introduction}

A large number of direct imaging surveys searching for substellar companions have been performed in recent years, yielding the first directly-imaged exoplanets (Bowler 2016). The efforts have been put into looking for these objects around young and nearby stars, where contrast ratios are more favorable. These discoveries reveal colors and spectral features that can indicate the composition of their atmospheres and their underlying physical properties (e.g., Barman et al. 2011; Currie et al. 2011; Faherty et al. 2016; Biller \& Bonnefoy 2018).

The Scorpius Centaurus (Sco-Cen) region is the nearest OB association ( $\sim 100-200 \mathrm{pc}$, de Zeeuw et al. 1999), and contains young stars ranging from hot and very massive O-type stars to free-floating substellar objects (Cook et al. 2017). It is thus an ideal place in which to search for young and low-mass objects, allowing a detailed study of stellar evolution and planet formation mechanisms (e.g. Preibisch et al. 2002; Preibisch \& Mamajek 2008; Luhman \& Mamajek 2012; Currie et al. 2015; Pecaut \& Mamajek 2016).

The large number of stars present in this association has been used to statistically constrain the age of each subregion by comparing stars of different masses to theoretical isochrones, which has shown an intricate star formation history (e.g., Pecaut \& Mamajek 2016). One of the most alluring results is the existence of a mass-dependent age trend, i.e., models yield younger ages for cooler low-mass stars compared to the corresponding massive population in the same subregion. For instance, the Upper Sco (USco) subregion would have a median age of about $5 \mathrm{Myr}$ if we consider only the pre-main sequence (PMS) K- and early M-type population, which is half of the usually adopted USco age of $10 \pm 3 \mathrm{Myr}$ from PMS G- and F-type stars. This discrepancy has also been similarly observed in other regions (e.g., Hillenbrand 1997; Bell et al. 2015; Herczeg \& Hillenbrand 2015). Two main explanations have been proposed to explain this fact; the effect of magnetic fields, which might inhibiting convection and slow down the contraction rate in low-mass PMS stars (Feiden 2016; Somers \& Stassun 2017), or an age spread within young clusters (Fang et al. 2017).

Here we present a SCExAO/CHARIS spectroscopic study of the USco HIP 79124 system encompassing the $J H K_{\mathrm{s}}$ near-IR bands $(1.1-2.4 \mu \mathrm{m})$. This young A-type star forms a triple system with two resolved low-mass companions at a very close projected separation. The outer companion, HIP $79124 \mathrm{C}$, was discovered at about $1^{\prime \prime}(\sim 137 \mathrm{AU})$ by AOassisted direct imaging surveys, first by Kouwenhoven et al. $(2005,2007)$ with ADONIS, which flagged it as a companion, and later by Lafreniere et al. (2014) with NIRI at the Gemini North telescope. Recently, an even more interesting discovery was the presence of an additional $\sim 135 M_{\text {Jup }}$ companion, HIP $79124 \mathrm{~B}$, interior to the $\mathrm{C}$ component, using aperture masking interferometry in $L^{\prime}$ band (Hinkley et al. 2015). This companion has also been imaged for the first time at a separation 
Table 1. HIP 79124 observing Log.

\begin{tabular}{|c|c|c|c|c|c|c|c|}
\hline $\begin{array}{l}\text { Date } \\
\text { (UT) }\end{array}$ & Telescope/Instrument & $\begin{array}{c}\text { Wavelength } \\
\text { (Filter) }\end{array}$ & Coronagraph & $\begin{array}{c}t_{\text {int }} \\
(\mathrm{sec})\end{array}$ & $N_{\text {images }}$ & $\begin{array}{l}\text { Collected PA } \\
(\text { deg })\end{array}$ & Mode \\
\hline 2017-07-15 & Subaru/CHARIS & $1.1-2.4 \mu \mathrm{m}$ & Lyot & $31 \times 1.475$ & 15 & 4.3 & IFS \\
\hline 2017-07-15 & Subaru/CHARIS & $1.1-2.4 \mu \mathrm{m}+\mathrm{ND}$ filter & - & $100 \times 0.2$ & 3 & 2.0 & IFS \\
\hline Archival Data & & & & & & & \\
\hline 2016-04-13 & Keck/NIRC2 & $L^{\prime}(3.8 \mu \mathrm{m})$ & Vortex & 30 & 26 & 18.8 & Imaging \\
\hline
\end{tabular}

Notes. The archival 2016 Keck/NIRC2 data was published in Serabyn et al. (2017).

of only $0.18^{\prime \prime}$ ( $\left.\sim 25 \mathrm{AU}\right)$ by Serabyn et al. (2017), using the new $L^{\prime}$ optical vortex coronagraph working alongside the AOassisted NIRC2/Keck camera.

In this work we will make use of our new low-resolution SCExAO/CHARIS observations together with archival Keck/NIRC2 $L^{\prime}$ photometry to analyze, for the first time, the spectral nature of the $\mathrm{B}$ and $\mathrm{C}$ companions. This study will improve the knowledge on the parameters of two low-mass stars in USco. Moreover, star-formation models favors a scenario where the HIP 79124 triple system is coeval, as the time difference of massive A-type formation compared to low-mass M-type objects seems to be securely below $1 \mathrm{Myr}$, even after including the accretion phase (Bate 2012). Binary systems in the Taurus association have also been shown to be more coeval than the region as a whole (Kraus \& Hillenbrand 2009). The likely coevality makes of this system a perfect laboratory to test whether the conventional isochronal models predict the same age for both the two low-mass companions and the massive and hot primary A-type star, or otherwise fail at reproducing the PMS evolution of low-mass stars.

\section{Observations and data reduction}

\subsection{SCEXAO/CHARIS data}

We observed the HIP 79124 system with the newly-established Coronagraphic High Angular Resolution Imaging Spectrograph (CHARIS) on 2017-07-15, located on the Nasmyth platform at the Subaru Telescope in Hawaii (Peters et al. 2012; Groff et al. 2013) coupled to the extreme adaptive optics system SCExAO (Jovanovic et al. 2015). We used the low-resolution $(R \sim 20)$ configuration of CHARIS, which covers the $J+H+K$ bands within a field of view (FoV) of $2.2^{\prime \prime} \times 2.2^{\prime \prime}$, and collected all data in pupil tracking/angular differential imaging mode (ADI, Marois et al. 2006). Both seeing conditions (e.g., $\left.\theta \sim 0.8^{\prime \prime}\right)$ and $\mathrm{AO}$ performance were slightly below average but sufficient to reveal both HIP 79124 companions without post-processing.

The observations consisted of two sequences. First, we acquired a first set of images using the Lyot coronagraph with a 217 mas diameter occulting spot to block the star, each of which consisting of 31 coadded $1.475 \mathrm{~s}$ exposures. After frame selection, our total integration time was $\sim 11$ minutes, covering a parallactic angle motion of $4.3 \mathrm{deg}$. As HIP $79124 \mathrm{C}$ was easily visible but possibly in the non-linear regime of the detector in a few spectral channels in the coronagraphic data, we acquired a second set of non-coronagraphic data using a neutral density filter to keep both $\mathrm{A}$ and $\mathrm{C}$ in the linear count regime totalling $60 \mathrm{~s}$ of integration time and covering a smaller parallactic angle motion of $\sim 2$ deg. Table 1 shows the observation log of the CHARIS data and the archival NIRC2/Keck data that we retrieve to complement our analysis (see Sect. 2.2).
For data cube extraction, we used the "least squares" method presented in the CHARIS Data Reduction Pipeline (CHARIS DRP; Brandt et al. 2017), yielding 22 images at wavelengths between $1.1-2.4 \mu \mathrm{m}$. An IDL-based CHARIS data reduction pipeline was used to perform the basic reduction processes, such as background subtraction, flat fielding and image registration (Currie et al. 2018a) following previous methods applied for broadband imaging data (e.g., Currie et al. 2011). To spectrophotometrically calibrate each data cube, we used an A0V spectral type from the Pickles (1998) stellar library, which is shown to be accurate despite issues with the library at other spectral types (Currie et al. 2018a) and is also adopted in the GPI Data Reduction Pipeline (Perrin et al. 2014). For the unsaturated data, the star's photometry is used directly for flux-calibration. For the coronagraphic data, satellite spots provided absolute spectrophotometic calibration (e.g., Currie et al. 2018b). We verified and fine-tuned our spectrophotometric calibration by 1) comparing the brightness of HIP $79124 \mathrm{C}$ in the saturated and unsaturated data and 2) correcting for signal loss due to the Lyot occulting spot $\left(\sim 92.5 \%\right.$ throughput at $\left.\rho \sim 0.18^{\prime \prime}\right)$.

\subsection{Keck/NIRC2 $L_{p}$ archival data}

The $L^{\prime}$ magnitude of the B component was already presented in Hinkley et al. (2015) and Serabyn et al. (2017); however, neither paper reported photometry for HIP $79124 \mathrm{C}$. To obtain it, we reduced the archival Keck/NIRC2 $L^{\prime}$ vortex data from June 2015 and April 2016 published in Serabyn et al. (2017), focusing on acquisition frames where the primary and $\mathrm{C}$ are both unsaturated. Basic processing followed previous steps employed for reducing NIRC2 $L^{\prime}$ archival data (Currie et al. 2011, 2018a), including a linearity correction, sky subtraction and distortion correction. The results from both epochs were consistent within error bars, and we adopted the smaller photometric uncertainty.

\subsection{SCEXAO/CHARIS detections}

In Fig. 1 we show the wavelength-collapsed image of HIP 79124 obtained from combining our coronagraphic exposures. Given that little field rotation was collected, we refrain from applying a point spread function (PSF)-subtraction algorithm that benefits from the ADI observing technique. Instead, we simply subtract a radial profile, which is sufficient to recover the B companion at a decent signal-to-noise ratio $(\mathrm{S} / \mathrm{N})$.

To compute the $\mathrm{S} / \mathrm{N}$ for both the $\mathrm{B}$ and $\mathrm{C}$ objects, we divide the convolved flux, measured at each companion's position, by the standard deviation of the convolved residual noise in concentric annuli at their same separation after excluding the signal from the companion (e.g., Thalmann et al. 2009; Currie et al. 2011), and corrected for finite sample sizes (Mawet et al. 2014). Our simple wavelength-collapsed radial profile-subtracted image yields $\mathrm{S} / \mathrm{N} \sim 9$ and $\sim 120$, respectively 


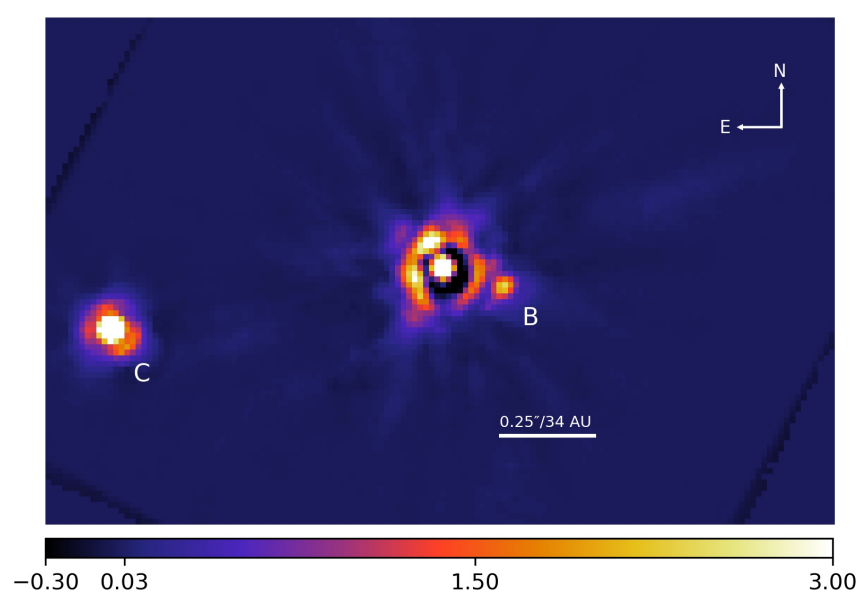

Fig. 1. Wavelength-collapsed $J H K_{\mathrm{s}}$ CHARIS image of the HIP 79124 stellar system. After subtracting a radial profile, both the B and C lowmass companions are clearly detected at a $\mathrm{S} / \mathrm{N}$ of $\sim 9$ and $\sim 120$, respectively. Another set of non-coronagraphic data was used to extract the spectrum of the $\mathrm{C}$ companion.

for B and C. As expected, the brightness and separation of the $\mathrm{C}$ companion provides a very strong detection in all individual channels. Although the situation is more complicated for the very close $\mathrm{B}$ object, SCExAO/CHARIS is able to identify it with a $\mathrm{S} / \mathrm{N}$ of $\sim 6-8$ for the shortest wavelengths in the data cube, peaking at $2-2.2 \mu \mathrm{m}$ with a $\mathrm{S} / \mathrm{N}$ of $\sim 11$.

From Fig. 1 we also obtain the astrometric position of the unsaturated companions with respect to the primary. We perform a Gaussian fit to extract the centroid of the point sources, whose error is estimated by dividing the FWHM of the candidate's PSF by its S/N (Thalmann et al. 2014). To this uncertainty, we add those from the plate scale of $16.2 \pm 0.1 \mathrm{mas} / \mathrm{spaxel}$ and true north orientation of $-2.20 \pm 0.27 \mathrm{deg}$ (Currie et al. 2018a). The inner companion is detected at a projected separation of $\rho=180 \pm 5$ mas $(\sim 25 \mathrm{AU})$ and a position angle of $252.9 \pm 1.6 \mathrm{deg}$. Within error bars, the angular separation is consistent with those obtained by Hinkley et al. (2015) in April 2010 and Serabyn et al. (2017) in April 2016. The position angle increases in time over these previous measurements, supporting evidence for counterclockwise orbital motion first found by Serabyn et al. (2017). We find C at $0.967 \pm 0.006^{\prime \prime}(\sim 132 \mathrm{AU})$ and $100.39 \pm 0.04 \mathrm{deg}$. Using the archival astrometric data from Lafreniere et al. (2014), we confirm that the C companion shares common proper motion with the primary star.

\section{Extracted CHARIS spectra and photometry}

\subsection{Spectrophotometry and reddening}

Figure 2 shows the CHARIS low-resolution extracted spectra for the HIP $79124 \mathrm{~B}$ and C companions in units of $\mathrm{Wm}^{-2} \mu \mathrm{m}^{-1}$. We carried out the extraction by defining an aperture of $0.5 \lambda / \mathrm{D}$ around the position of each companion in the wavelengthcollapsed image.

The B and C spectra present very similar features. They show a downward trend in flux toward redder wavelengths, except for a peak or plateau in the $H$ band region $(\lambda \sim 1.65 \mu \mathrm{m})$. The $\mathrm{C}$ companion is brighter and detected with a very high $\mathrm{S} / \mathrm{N}$, which generate very small error bars during the extraction. In this case, the error bars are dominated by the absolute calibration uncertainty.
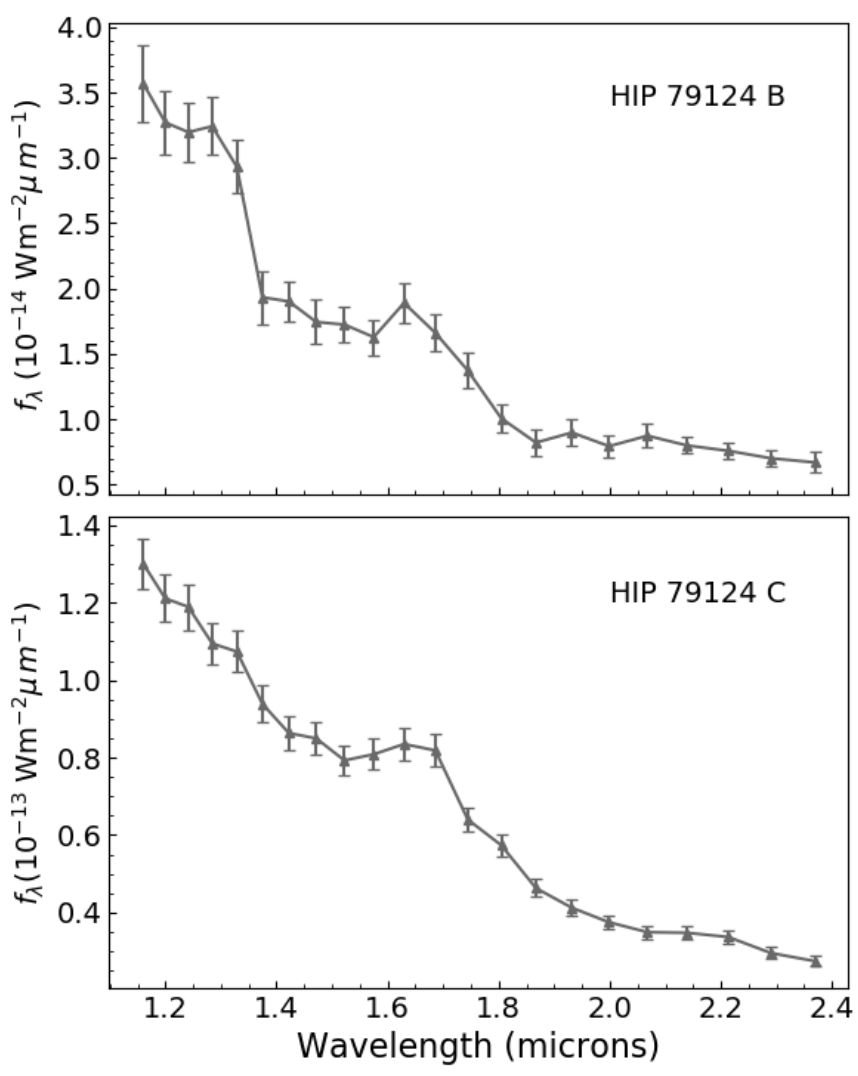

Fig. 2. SCExAO/CHARIS $J H K_{\mathrm{s}}$ spectra of the HIP $79124 \mathrm{~B}$ and C companions. The flux has been calibrated with an A0V template from Pickles (1998) and dereddened by $A_{V}=0.82$.

The photometry of the triple system is presented in Tables 2 and 3. We convolved each spectrum with the Mauna Kea Observatory $(\mathrm{MKO}) J H K_{s}$ passbands functions, previously binned down to CHARIS' low-resolution mode. We note that the photometric values for the $\mathrm{C}$ companion are very close to the ones given by Kouwenhoven et al. (2007), although the $J$ band photometry is slightly brighter.

The $J H K_{\mathrm{s}}$ 2MASS magnitudes of the primary were converted to the MKO system by means of the transformation equations in Carpenter (2001). We checked that the C companion does not affect the 2MASS photometry significantly, as the difference between including or not including the flux of the companion in the magnitude of the primary is within $2 \sigma$ of the primary error bar in the $K_{s}$ and $L^{\prime}$ bands.

We also estimated the extinction for our target using the intrinsic color of nearby dwarf A0-type stars with negligible extinction (Pecaut \& Mamajek 2013). We computed $E(B-V)$, $E(V-J), E(V-H)$ and $E(V-K \mathrm{~s})$. Taking $\mathrm{R}_{\mathrm{V}}=3.1$ as extinction law, and the extinction coefficients from Fiorucci \& Munari (2003), we obtain a median $A_{V}=0.82 \pm 0.05 \mathrm{mag}, A_{J}=$ $0.23 \pm 0.02 \mathrm{mag}, A_{H}=0.136 \pm 0.008 \mathrm{mag}$ and $A_{K s}=0.088 \pm$ $0.005 \mathrm{mag}$ from the four different colors and adopt the scatter as uncertainties. This $A_{V}$ value is in agreement within errorbars with the values previously obtained by Pecaut et al. (2012) and Hinkley et al. (2015). No extinction is assumed in $L^{\prime}$, and we use these values to derive dereddened absolute magnitudes for a Gaia-DR2 distance of $137.0 \pm 1.2$ pc (Lindegren et al. 2018). We dereddened the spectra of the $\mathrm{B}$ and $\mathrm{C}$ companions by fitting a second-order polynomial to the extinction coefficients, obtaining a coefficient for each of the CHARIS wavelength 
Table 2. Photometry of HIP 79124 B.

\begin{tabular}{llcccccc}
\hline \hline UT Date & Telescope/Camera & Filter & $\begin{array}{c}\text { Primary } \\
(\mathrm{mag})\end{array}$ & $\begin{array}{c}\text { Companion } \\
(\Delta \mathrm{mag})\end{array}$ & $\begin{array}{c}\text { Apparent mag } \\
(\mathrm{mag})\end{array}$ & $\begin{array}{c}\text { Absolute mag } \\
(\text { dereddened })\end{array}$ & Ref. \\
\hline $2016-04-13$ & Keck/NIRC2 & $\mathrm{W} 1 / L^{\prime}$ & $6.96 \pm 0.04^{a}$ & $4.25 \pm 0.14^{c}$ & $11.16 \pm 0.11$ & $5.48 \pm 0.11$ & 2,3 \\
$2017-07-15$ & SCExAO/CHARIS & $J$ & $7.17 \pm 0.03^{b}$ & $5.48 \pm 0.13$ & $12.65 \pm 0.13$ & $6.73 \pm 0.13$ & 1 \\
$2017-07-15$ & SCExAO/CHARIS & $H$ & $7.00 \pm 0.05^{b}$ & $5.26 \pm 0.15$ & $12.26 \pm 0.15$ & $6.44 \pm 0.15$ & 1 \\
$2017-07-15$ & SCExAO/CHARIS & $K s$ & $7.003 \pm 0.018^{b}$ & $4.92 \pm 0.15$ & $11.93 \pm 0.15$ & $6.15 \pm 0.15$ & 1 \\
\hline
\end{tabular}

Notes. ${ }^{(a)}$ From the WISE W1 channel (Cutri et al. 2012). ${ }^{(b)}$ From the 2MASS catalog (Cutri et al. 2003) converted to the MKO passbands via Carpenter (2001). ${ }^{(c)}$ Mean of the magnitude contrast published in Hinkley et al. (2015) and Serabyn et al. (2017).

References. (1) This paper; (2) Serabyn et al. (2017); (3) Hinkley et al. (2015).

Table 3. Photometry of HIP 79124 C.

\begin{tabular}{llcccccc}
\hline \hline UT Date & Telescope/Camera & Filter & $\begin{array}{c}\text { Primary } \\
(\mathrm{mag})\end{array}$ & $\begin{array}{c}\text { Companion } \\
(\Delta \mathrm{mag})\end{array}$ & $\begin{array}{c}\text { Apparent mag } \\
(\mathrm{mag})\end{array}$ & $\begin{array}{c}\text { Absolute mag } \\
(\text { dereddened })\end{array}$ & Ref. \\
\hline $2016-04-13$ & Keck/NIRC2 & $\mathrm{W} 1 / L^{\prime}$ & $6.96 \pm 0.04^{a}$ & $2.98 \pm 0.03$ & $9.94 \pm 0.05$ & $4.26 \pm 0.05$ & 1,2 \\
$2017-07-15$ & SCExAO/CHARIS & $J$ & $7.17 \pm 0.03^{b}$ & $4.08 \pm 0.01$ & $11.27 \pm 0.05$ & $5.36 \pm 0.05$ & 1 \\
$2017-07-15$ & SCExAO/CHARIS & $H$ & $7.00 \pm 0.05^{b}$ & $3.54 \pm 0.01$ & $10.57 \pm 0.05$ & $4.75 \pm 0.05$ & 1 \\
$2017-07-15$ & SCExAO/CHARIS & $K s$ & $7.003 \pm 0.018^{b}$ & $3.344 \pm 0.01$ & $10.35 \pm 0.03$ & $4.58 \pm 0.05$ & 1 \\
\hline
\end{tabular}

Notes. ${ }^{(a)}$ From the WISE W1 channel (Cutri et al. 2012). ${ }^{(b)}$ From the 2MASS catalog (Cutri et al. 2003) converted to the MKO passbands via Carpenter (2001).

References. (1) This paper; (2) Serabyn et al. (2017).

channels, which we used to correct for the reddening in our spectra.

\subsection{Correlated noise}

When fitting integral field spectrograph (IFS) data to model spectra, it is of vital importance to consider the effect of covariances to properly retrieve atmospheric parameters. As shown by Greco \& Brandt (2016), in high-contrast imaging it is not easy to understand the impact that the data analysis techniques have on the extracted spectrum. Following their work, we first compute the spectral correlation at HIP 79124 B's and C's positions in the collapsed image. To this aim, we normalize each channel by its standard deviation profile and, for each pair of CHARIS wavelengths $i$ and $j$, compute its correlation $\Psi_{\mathrm{ij}}$ in a $2 \lambda / \mathrm{D}$-wide ring centered on the companion:

$\Psi_{i j} \equiv \frac{<I_{i} I_{j}>}{\sqrt{<I_{i}^{2}><I_{j}^{2}>}}$,

where $I_{\mathrm{i}}$ and $I_{\mathrm{j}}$ are the intensities at wavelengths $i$ and $j$, respectively, averaged over all the spatial locations within the annulus, and masking the $2 \lambda / \mathrm{D}$ region around the companion.

The correlation matrices for both companions are shown in Fig. 3. The noise is very much uncorrelated at the position of HIP $79124 \mathrm{C}\left(\sim 1^{\prime \prime}\right)$, with a nearly constant value of $\sim 0.2$ except for $i=j$. This might be due to the fact that we used the unsaturated raw image for spectral extraction of this outer companion. For the inner companion at $\sim 0.18^{\prime \prime}$, HIP $79124 \mathrm{~B}$, the noise is highly correlated and the correlation varies between channels.

The populated $\Psi_{\mathrm{ij}}$ vs $\lambda_{\mathrm{ij}}$ distribution can be fit using a Levenberg-Marquardt minimization to the functional form

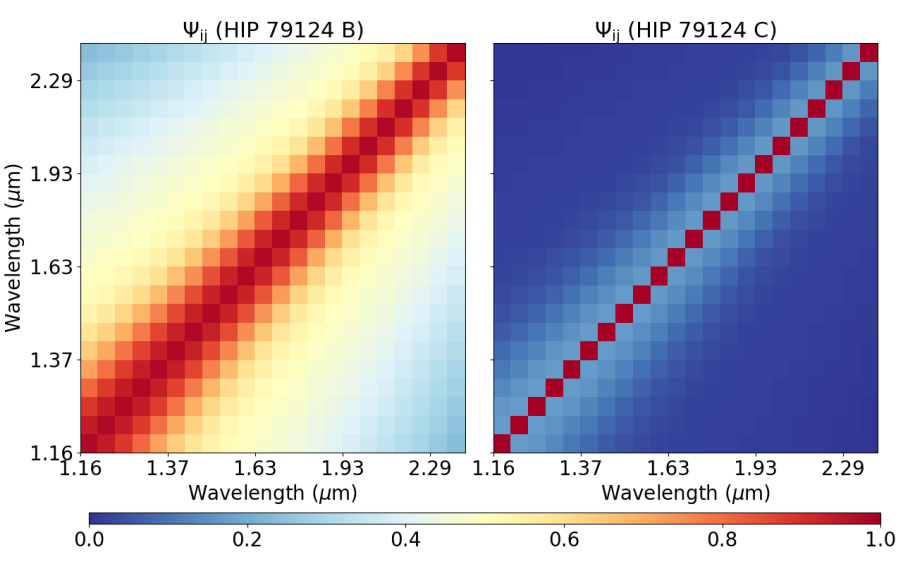

Fig. 3. Correlation matrices calculated via Eq. (1) for the B (left panel) and $\mathrm{C}$ (right panel) companions to HIP $79124 \mathrm{~A}$. The reduction process of the IFS data introduced spectrally correlated noise in the extracted spectra. The small projected separation at which B is located makes the noise to be highly-correlated, as the speckles from the primary PSF add flux density within the companion's PSF over different channels.

described in Greco \& Brandt (2016):

$$
\begin{gathered}
\Psi_{i j} \approx A_{\rho} \exp \left[-\frac{1}{2}\left(\frac{\rho}{\sigma_{\rho}} \frac{\lambda_{i}-\lambda_{j}}{\lambda_{c}}\right)^{2}\right]+ \\
A_{\lambda} \exp \left[-\frac{1}{2}\left(\frac{1}{\sigma_{\lambda}} \frac{\lambda_{i}-\lambda_{j}}{\lambda_{c}}\right)^{2}\right]+ \\
A_{\delta} \delta_{i j},
\end{gathered}
$$

where the spatially and spectrally correlated noise have amplitudes $A_{\rho}$ and $A_{\lambda}$, and characteristic lengths $\sigma_{\rho}$ and $\sigma_{\lambda}$, respectively. $A_{\delta}$ is the amplitude of the uncorrelated term. 
We redo this minimization several times at separations bracketing the companions, masking them and any residual satellite spot light. In the case of the B companion, it appears that the spectral covariance is not optimally described by this functional form, as there are two secondary peaks in $\Psi_{i j}$ that flank the main peak at $i=j$. Comparing plots for different separations, these peaks move out from $\rho\left(\lambda_{i}-\lambda_{j}\right) /\left(\lambda_{c}\right) \sim 1.5-2.5$, as we go from separations of $\rho=4-6$ in units of $\lambda_{\mathrm{c}} / \mathrm{D}$. The peaks disappear at $\rho=3$ and $\rho>7$. In any case, our best fit for the B companion at its separation of $\rho=4.25$, is $A_{\rho}=0.42, A_{\lambda}=0.54$, $A_{\delta}=0.02, \sigma_{\rho}=0.34$ and $\sigma_{\lambda}=0.57$. This shows that at the location of the $\mathrm{B}$ object, the uncorrelated component $A_{\delta}$ is very much negligible compared to the correlated noise, which is dominant at small separations where speckle noise is not well eliminated. In the case of the $\mathrm{C}$ companion the uncorrelated amplitude dominates at $\rho=4.25$, with $A_{\rho}=0.12, A_{\lambda}=0.21, A_{\delta}=0.65, \sigma_{\rho}=2.15$ and $\sigma_{\lambda}=1.69$. As we extracted the $\mathrm{C}$ spectrum from the unsaturated dataset, the resulting correlation length is large perhaps because the background has not been well flattened from a leastsquares PSF subtraction.

\section{HIP 79124 empirical constraints}

\subsection{Spectral types of HIP 79124 B and C}

Although we have adopted an A0V spectral type for the primary star from Houk \& Smith-Moore (1988), the spectral type of the companions has never been assessed. To get a good estimation, we first compare the CHARIS near-IR spectrum of HIP 79124 $\mathrm{B}$ and $\mathrm{C}$ to libraries of ML empirical objects in young moving groups. Then, we adopt a second approach, refining the classification by comparing the observed HIP 79124 spectra to a set of $\sim 10$ Myr-old M-type standard spectra.

\subsubsection{Comparison to empirical ML spectra}

As HIP 79124 is a member of the USco young star-forming region with an age of only $10 \pm 3 \mathrm{Myr}$ (Pecaut \& Mamajek 2016), we decide to use libraries of young objects, which are warmer than their field counterpart (and have earlier spectral type) for a given mass, and are still contracting, presenting inflated radii and thus low surface gravities that affect their spectra. An indication of youth in low-resolution near-IR spectra is the triangular $H$-band continuum shape, which becomes less pronounced as one moves from very low $(\delta)$ to low $(\gamma)$ and intermediate-gravity $(\beta)$ late $M$ - and $L$-type dwarfs. In comparison, field objects tend to show a plateau (e.g., Allers \& Liu 2013). Other indicators exist also in the $J$ and $K$ bands, such as FeH absorption (McLean et al. 2003) or the $K$-band slope (see the $\mathrm{H}_{2}(K)$ index, Canty et al. 2013; Currie et al. 2014a).

We mainly adopt the young population of the Montreal Spectral library ${ }^{1}$ as the source for comparison spectra. These objects are members of nearby young moving groups $(\leq 120 \mathrm{Myr})$, with spectral types in the $M L T$ range and $\delta, \gamma$ and $\beta$ gravities. We consider only high $\mathrm{S} / \mathrm{N}$ objects, leaving out those with median uncertainties larger than $5 \%$ of the median flux value. These spectra come mainly from Gagne et al. (2015) and were obtained with several instruments, such as Flamingos - 2 (Eikenberry et al. 2004) and SpeX (Rayner et al. 2003). Also, we include the near-IR Bonnefoy et al. (2014) VLT/SINFONI library of young dwarfs in the $M-L$ transition (M8.5-L4).

\footnotetext{
1 https://jgagneastro.wordpress.com/ the-montreal-spectral-library/
}
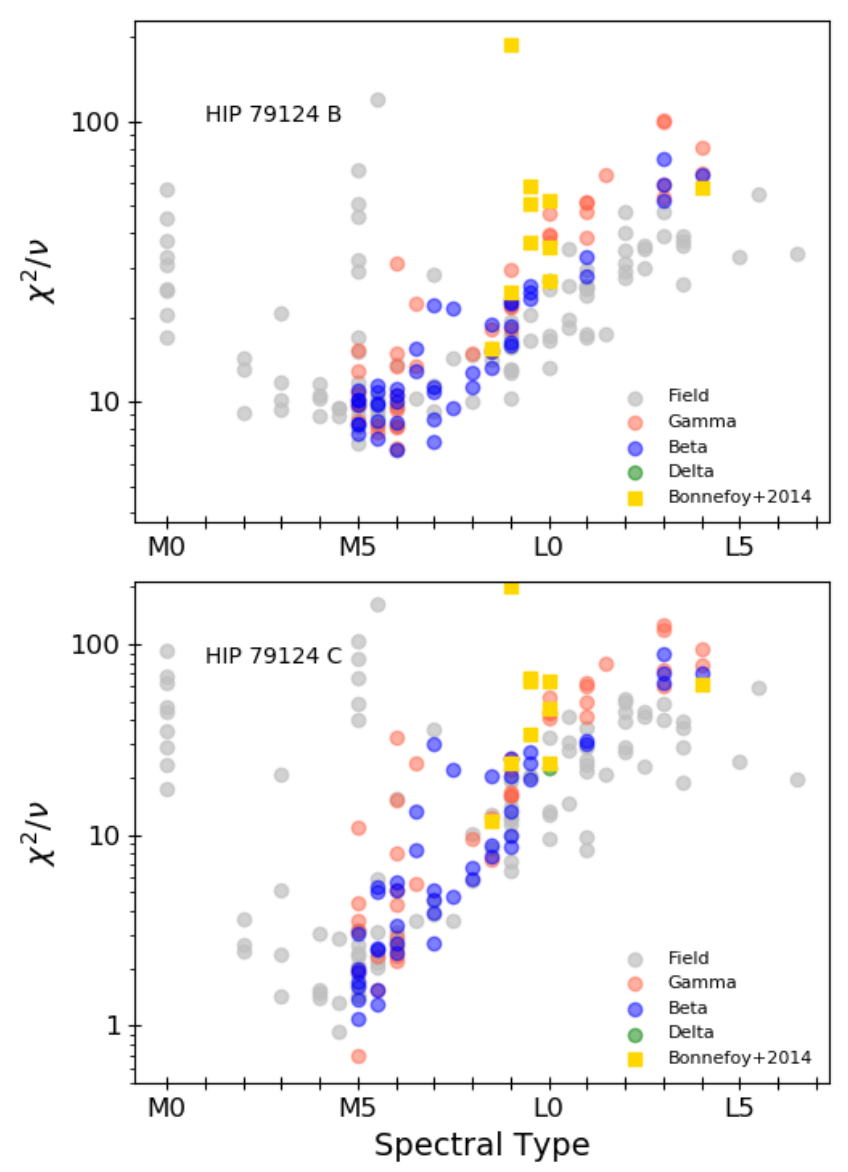

Fig. 4. Normalized $\chi^{2}$ for the B and C CHARIS spectra as compared to the empirical objects of the Montreal (Gagne et al. 2015) and BonnefoyVLT/SINFONI (Bonnefoy et al. 2014) libraries.

In the chi-square goodness of fit statistic we incorporate the correlated errors via the covariance matrix $C$ :

$\chi_{k}^{2}=\left(S-f_{k} F_{k}\right)^{T} C_{k}^{-1}\left(S-f_{k} F_{k}\right)$,

where $S$ is the set of observed spectral values by CHARIS. To create a similar set of these flux values for each of the comparison spectra, we first smooth the empirical model to the CHARIS low resolution. Then, for each of the 22 CHARIS channels spanning the $1.1-2.4 \mu \mathrm{m}$ range, we estimate a flux value via interpolation. In this way we create a vector $F$ for each empirical $k$ object that will be compared to $S$. The comparison object is multiplied by a constant $f_{\mathrm{k}}$, which was introduced in Cushing et al. (2008), that minimizes $\chi^{2}$ and accounts for the distance difference between the observed CHARIS spectrum and the empirical object. We focus on regions covering the major $J H K$ passbands, avoiding strong telluric absorption, leaving us with 15 out of the 22 channels that will be used for the comparison.

To construct the $22 \times 22$ covariance matrix $C$, we use the correlation matrix $\Psi_{\mathrm{ij}}$ presented in Fig. 3 for the B and C companions. The off-diagonal elements of $\Psi_{\mathrm{ij}}$ are multiplied by the uncertainty in the flux of the corresponding $i j$ channels. For the outer companion, the error bars from the extracted spectrum are on the order of $0.1 \%$, and the absolute calibration uncertainty dominates, with values $2-4 \%$ of the observed flux. The on-diagonal are also affected by the uncertainties on the uncorrelated comparison spectrum, which we add in quadrature to the correlated errors. 

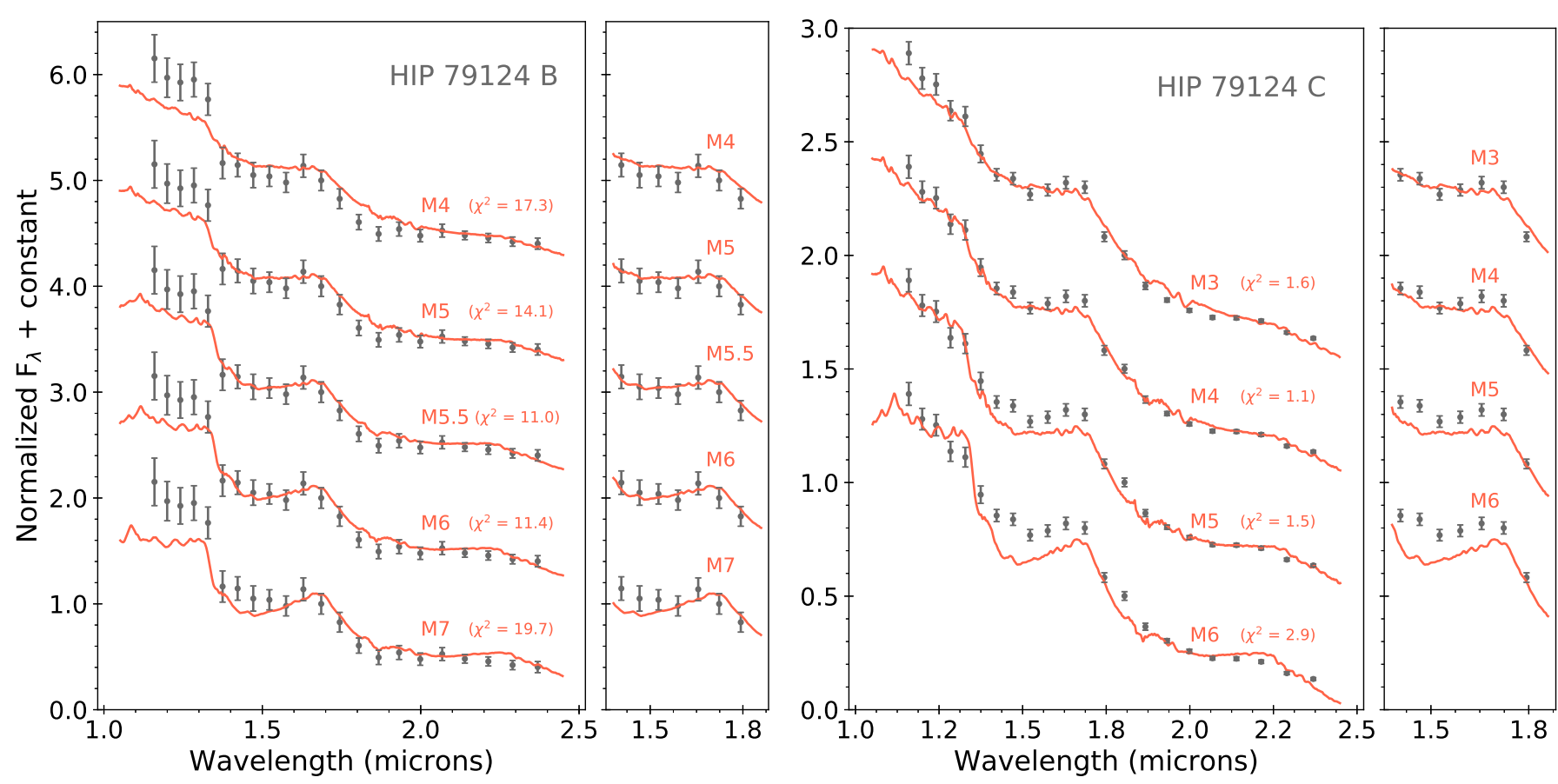

Fig. 5. Spectral fits of the B (left panel) and C (right panel) companions to the old population ( 10 Myr) of standard spectra from Luhman et al. (2017). Our companions have been corrected for reddening assuming the same extinction as the primary star (see Sect. 3.1). To compute the $\chi^{2}$ per degree of freedom, we refrain from using spectral regions affected by tellurics. The panels on the right of each $\mathrm{B}$ and $\mathrm{C}$ comparison is an $H$-band zoom-in, which is more affected by the gravity of the object.

Figure 4 shows the resulting $\chi^{2}$ per degree of freedom for the Montreal and Bonnefoy libraries, filtered out for objects with a signal to noise lower than $5 \%$. For the outer $\mathrm{C}$ companion, M3-M5.5 objects fall within the $\Delta \chi^{2} 95 \%$ confidence level for 15 degrees of freedom. Low-gravity comparison objects with spectral types earlier than M5 are unavailable. In any case, the best-fit empirical spectrum is the low-gravity M5 $\gamma$ 2MASS J0259-4232 object in the 20-40 Myr-old Columba association (Rodriguez et al. 2013).

The situation for the inner companion is however more complicated, as the large correlation among the channels yield $\chi^{2}$ results that are higher than in the non-correlated scenario. It also broadens the $\Delta \chi^{2}$ space of good-fit models (see Greco \& Brandt 2016), which we clearly see in the bow-shape distribution for HIP 79124 B. In this case none of the empirical spectra fall within the $95 \%$ confidence region. For this reason, we adopt a confidence interval of $\Delta \chi^{2}<\sqrt{2 \chi^{2} \text { min }}$ (e.g., Thalmann et al. 2013), which encompasses spectral types in the range M5-M7.

\subsubsection{Comparison to M-type composite standard spectra}

Here, we adopt the dereddened near-IR standard spectra constructed by Luhman et al. (2017), where they combine several optical spectra for each subtype in the $\mathrm{M}$ spectral region. These resulting templates are representative of young associations and can be used for classifying the spectral type of young stars. We take the set of templates produced from a population of objects members of both USco and the TW Hya association (TWA). TWA is located at $\sim 50 \mathrm{pc}$ and, like USco, it has an estimated age of $\sim 10$ Myr (Webb et al. 1999; Mamajek 2005; Donaldson et al. 2016). Following the same procedure as for the library of empirical spectra, we compare the spectrum of HIP 79124 B and C with this set of M-type standard spectra. The results are shown in Fig. 5. The outer companion is well reproduced in the $J H K$ bands by the M3-M5 spectral standards. The best-fit falls in the M4 type, which is particularly successful at duplicating the $H$ band part of the spectrum. The B companion finds a clear minimum in the M5.5-M6 spectral type regime, where both the $H K$ are very well matched.

The $J$ passband of the CHARIS spectrum of B is slightly brighter than the standard spectra, and it is so consistently for all the spectral types fitting well the $H K$ bands. This might be due to speckle contamination at the shortest wavelengths by the primary star, given the small projected distance at which HIP 79124 B is located. The Strehl ratio (for a given residual wavefront error) and $\mathrm{S} / \mathrm{N}$ are also lower at these wavelengths, which ultimately may lead to a suboptimal spectral extraction. Another possibility is that we overestimated the extinction of HIP 79124 $\mathrm{B}$, as in some cases components of young multiple systems have different reddening factors (Kevin Luhman, priv. comm.). We thus adopt a series of different $A_{\mathrm{V}}$ and compute their corresponding $A_{\mathrm{J}}, A_{\mathrm{H}}$ and $A_{\mathrm{K}_{\mathrm{s}}}$ extinction factors using the relations from Fiorucci \& Munari (2003). In the upper panel of Fig. 6 we show the best-fit standard spectrum for each $A_{\mathrm{V}}$ value for both HIP $79124 \mathrm{~B}$ and $\mathrm{C}$. In the lower panel we show the best-fit spectrum for the combined extinction-spectral type set of parameters. The extinction that minimizes the residuals for the outer companion seems to agree quite well with the derived reddening factor of the primary star $\left(A_{\mathrm{V}}=0.82\right)$. However, a extinction-free scenario for $\mathrm{B}$ reproduces the SCExAO/CHARIS data much better. As the spectral types are consistent with those obtained in Fig. 5, for the rest of the calculations we will assume that the computed reddening for the primary star applies to the triple system.

\subsubsection{Final adopted spectral types}

Based on the comparison with empirical spectra from the Montreal library and with the Luhman et al. (2017) standard 


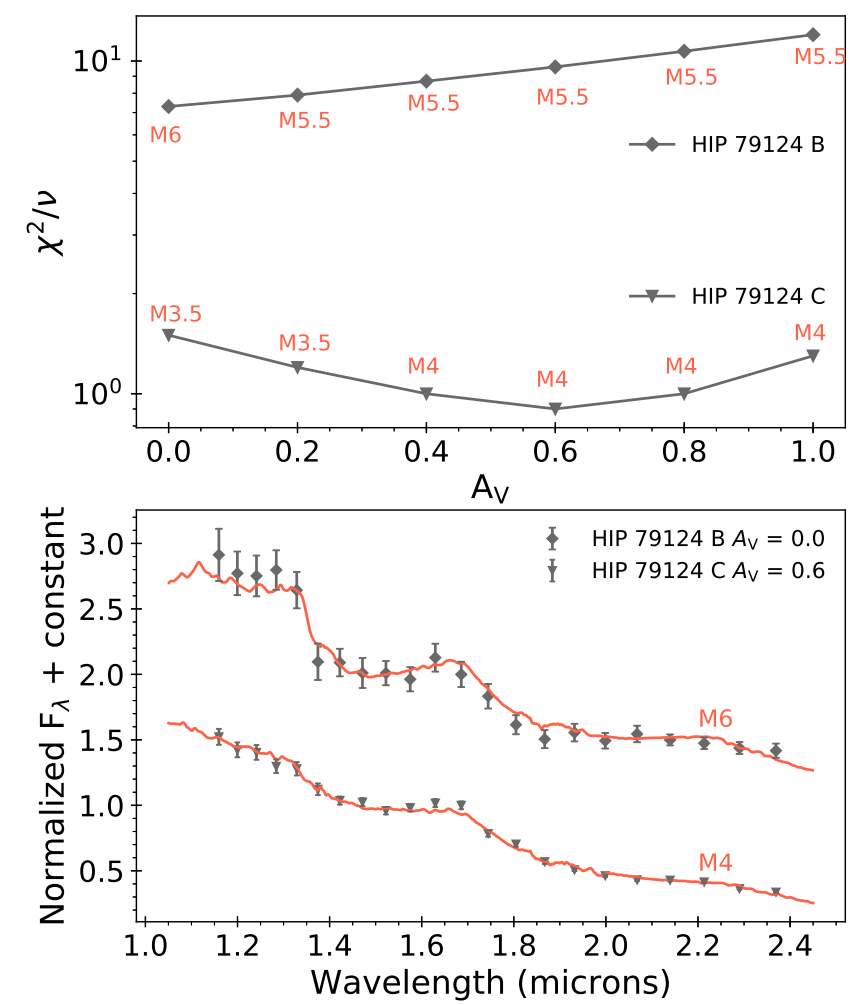

Fig. 6. Top panel: normalized best-fit $\chi^{2}$ for the $\mathrm{B}$ and $\mathrm{C}$ SCExAO/CHARIS spectra for a range of different extinctions. The spectral type that corresponds to the best-fit for each individual extinction value is indicated next to each data point. Bottom panel: SCExAO/CHARIS B and C spectra compared to the best-fit standard from Luhman et al. (2017). These SCExAO/CHARIS spectra have been corrected for the extinction value that better minimized the residuals as found in the top panel.

templates, here we summarize the final spectral types and uncertainties that we adopt for the companions. For the $\mathrm{C}$ component, from Sect. 4.1.1 we obtained a 95\% confidence level for M3M5.5 spectral types, with a dearth of young objects in the Montreal library for spectral types earlier than M5, and an M4 bestfit from Sect. 4.1.2. We adopt a final spectral type of M4 \pm 0.5 , which accurately reproduces the $J H K$ passbands of $\sim 10 \mathrm{Myr}$ standard spectra (see Fig. 5). For the inner B companion we adopt a spectral type of M6 \pm 0.5 . The Montreal objects favor an M6-type, and a minimum at M5.5-M6 is found using the standard templates.

\subsection{HR diagram: age of the HIP 79124 triple system}

Once we have an estimation of the spectral type for each object in the triple system, we place its members on a HertzsprungRussell (HR) diagram to compare their position with theoretical models. This will hopefully allow us to constrain the age of the coeval system using such a diverse range of masses.

We estimate effective temperatures $\left(T_{\text {eff }}\right)$ and bolometric corrections (BC) from two different sources in the literature of young objects; Kraus \& Hillenbrand (2007) presented a set of spectral type models optimized with empirical data from the open cluster Praesepe, with an age of $600 \mathrm{Myr}$. More recently, Pecaut \& Mamajek (2013) used young moving group (5-30 Myr) members to also build a $T_{\text {eff }}$ scale by comparing their spectral energy distribution to BT-Settl atmospheric models (Allard et al. 2012), covering spectral types down to M5.

\subsubsection{Temperature and luminosity of the companions}

The estimated M4 \pm 0.5 spectral type for the $\mathrm{C}$ companion corresponds to a $T_{\text {eff }}=3160 \pm 140 \mathrm{~K}$ and $\mathrm{BC}_{J}=1.91 \pm 0.05 \mathrm{mag}$ from the table of young objects in Pecaut \& Mamajek (2013). From the SCExAO/CHARIS dereddened absolute magnitude in $J$ band shown in Table 3, using the $\mathrm{BC}_{\mathrm{J}}$ value we get a $\mathrm{M}_{\mathrm{BOL}}(\mathrm{C})=7.27 \pm 0.07 \mathrm{mag}$, which translates into a luminosity of $\log \left(L(\mathrm{C}) / L_{\odot}\right)=-1.01 \pm 0.03 \mathrm{dex}$ for a solar absolute magnitude of 4.755 mag.

Similarly for HIP $79124 \mathrm{~B}$, we obtain a $T_{\text {eff }}=2840 \pm 90 \mathrm{~K}$ from Kraus \& Hillenbrand (2007). Pecaut \& Mamajek (2013) do not count with values for young stars beyond M5, but the error bar encompasses their young M5 $T_{\text {eff }}$, and a spectral type later than M6.5 is hardly a good fit to the data, as seen in Sect. 4.1.2. Given that no bolometric color correction is available for such a late spectral type in Pecaut \& Mamajek (2013), and that there is the possibility of some contamination from the primary star at short wavelengths, we obtain a $\mathrm{BC}_{K}=3.03 \pm 0.13$ mag from Golimowski et al. (2004) for the best-fit field M6 dwarf. That leads to $\mathrm{M}_{\mathrm{BOL}}(\mathrm{B})=9.2 \pm 0.2 \mathrm{mag}$ and $\log \left(L(\mathrm{~B}) / L_{\odot}\right)=-1.77 \pm$ 0.08 dex.

\subsubsection{Temperature and luminosity of the host star}

In this case, we take two different approaches. First, as done for the B and C companions, from Pecaut \& Mamajek (2013) we can obtain a $T_{\text {eff }}=9700 \pm 700 \mathrm{~K}$ with an uncertainty of one subclass in the spectral type. As we have a well defined reddening factor in the $V$ band, we use it to calculate a bolometric magnitude $\mathrm{M}_{\mathrm{BOL}}(\mathrm{A})=1.1 \pm 0.2$. We adopted the $V$ apparent magnitude from Hinkley et al. (2015), the GaiaDR2 distance (Lindegren et al. 2018) and $\mathrm{BC}_{V}$ for A0 stars in Pecaut \& Mamajek (2013). This value is compatible within error bars with the result presented in Hinkley et al. (2015), although our computation is slightly brighter, as we have adopted the updated Gaia-DR2 distance. This magnitude corresponds to $\log \left(L(\mathrm{~A}) / L_{\odot}\right)=1.46 \pm 0.08 \mathrm{dex}$.

On the other hand, we attempt to refine these values by constructing the spectral energy distribution of HIP 79124 A from the data in the literature (see Table 4). We deredden the observed photometry by using a second-order polynomial fit to the values derived in Sect. 3.1, and refrain from using photometric points at wavelengths $\geq 10 \mu \mathrm{m}$, as they might be affected by the two low-mass companions. We then fit BT-Settl models (Allard et al. 2012) with $\log g=4.5 \mathrm{dex}$ and $[M / H]=0$, with solar reference abundances from Caffau et al. (2011), to the data via the $\mathrm{G}$ goodness-of-fit statistic presented in Cushing et al. (2008), which accounts for the individual filter widths. As the reddening factor for the entire wavelength range is uncertain (especially for the shortest wavelengths), we simply adopt an error bar of 5\% the flux of each passband. The results are presented in Fig. 7. There is a minimum at $9200 \mathrm{~K}$. Well-fitting models are again taken if their $G$ values are smaller than $G_{\min }+\sqrt{2 G_{\min }}$. That signifies an uncertainty of $600 \mathrm{~K}$, probably due to the absence of photometric values with $\lambda<0.36 \mu \mathrm{m}$ and the effect of reddening. On the other hand, integrating the best-fit BT-Settl spectrum of $T_{\text {eff }}=9200 \pm 600 \mathrm{~K}$ and using the Gaia-DR2 distance, we obtain $\log \left(L(\mathrm{~A}) / L_{\odot}\right)=1.40 \pm 0.07 \mathrm{dex}$

The two different approaches are clearly consistent with each other within error bars. To place HIP 79124 A in a HR dia- 
Table 4. HIP 79124, a SED observations.

\begin{tabular}{lllc}
\hline \hline Band & $\begin{array}{l}\text { Wavelength } \\
\pm \text { bandwidth }\end{array}$ & $\begin{array}{l}\text { Flux } \\
\left(10^{-10} \mathrm{erg} \mathrm{s}^{-1} \mathrm{~cm}^{-2} \mu \mathrm{m}^{-1}\right)\end{array}$ & Reference \\
\hline$U$ & $0.3620 \pm 0.1380$ & $242.469 \pm 11.628$ & $(1,2)$ \\
$B$ & $0.4412 \pm 0.1816$ & $399.360 \pm 19.153$ & $(1,2)$ \\
$G_{\mathrm{bp}}$ & $0.5050 \pm 0.2347$ & $303.500 \pm 0.376$ & $(3,4)$ \\
$V$ & $0.5529 \pm 0.1129$ & $290.463 \pm 6.965$ & $(1,2)$ \\
$G$ & $0.6230 \pm 0.4183$ & $209.466 \pm 0.077$ & $(3,4)$ \\
$G_{\text {rp }}$ & $0.7730 \pm 0.2757$ & $130.538 \pm 0.181$ & $(3,4)$ \\
$J$ & $1.2603 \pm 0.2095$ & $41.282 \pm 1.980$ & $(1,5)$ \\
$H$ & $1.6652 \pm 0.1362$ & $17.389 \pm 1.501$ & $(1,5)$ \\
$K$ & $2.2094 \pm 0.2142$ & $6.287 \pm 0.271$ & $(1,5)$ \\
$W 1$ & $3.350 \pm 0.660$ & $1.347 \pm 0.045$ & $(6)$ \\
IRAC 2 & $4.4930 \pm 1.0200$ & $0.443 \pm 0.005$ & $(7,8)$ \\
$W 2$ & $4.600 \pm 1.040$ & $0.398 \pm 0.007$ & $(6)$ \\
IRAC 4 & $7.8720 \pm 2.8810$ & $0.0499 \pm 0.0004$ & $(7,8)$ \\
\hline
\end{tabular}

References. (1) Mann \& von Braun (2015); (2) Myers et al. (2015); (3) Jordi et al. (2010); (4) Gaia Collaboration (2018); (5) Cutri et al. (2003); (6) Cutri et al. (2012); (7) Quijada et al. (2004); (8) Carpenter et al. (2006)

gram, we adopt the mean of the resulting values, and their scatter as uncertainty. In this way, we consider for the primary a $T_{\text {eff }}=9450 \pm 250 \mathrm{~K}$ and $\log \left(L(\mathrm{~A}) / L_{\odot}\right)=1.43 \pm 0.03 \mathrm{dex}$ for HIP 79124 A.

\subsubsection{HR diagrams}

The HIP 79124 system includes one high-mass star, just arrived (or about to) in the main sequence, and two low-mass premain sequence companions contracting along a Hayashi track (Siess et al. 2000; Pecaut et al. 2012). The different physical processes occurring on these objects might pose difficulties to the pre-main sequence evolution models, which (if accurate) should be expected to yield a common age estimate for the entire system. For the similar, albeit older, HD 1160 triple system studied by Garcia et al. (2017), the primary A-type star was on/just beginning to evolve off the main sequence and its two low-mass (M star) companions were closer to the main sequence. In this case, isochrone comparisons for the primary yielded younger age estimates than those for the two low-mass companions. Our study allows a similar analysis at young ages.

Figure 8 shows the luminosity- $T_{\text {eff }}$ diagram of the HIP 79124 triple system, where the objects are compared with evolutionary models at different ages. We use a different set of solar-metallicity evolutionary tracks to derive a range of wellfitting ages: the MESA Isochrones \& Stellar Tracks (MIST, Paxton et al. 2011, 2013, 2015; Choi et al. 2016; Dotter 2016) for the massive primary star and HIP 79124 C; the PARSECCOLIBRI stellar isochrones (Marigo et al. 2017) encompassing the triple system; both the original Dartmouth Stellar Evolution isochrones (Dotter et al. 2008) and those accounting for magnetic inhibition of convection ${ }^{2}$, which should be reliable for systems that are approximately $10 \mathrm{Myr}$, as the surface magnetic field strengths in those models were tuned for modeling $\sim 10 \mathrm{Myr}$ systems (Feiden 2016); and the BHAC15 Baraffe et al. (2015) evolutionary tracks for low-mass objects for the B and C companions.

\footnotetext{
2 http://github.com/gfeiden/MagneticUpperSco/
}
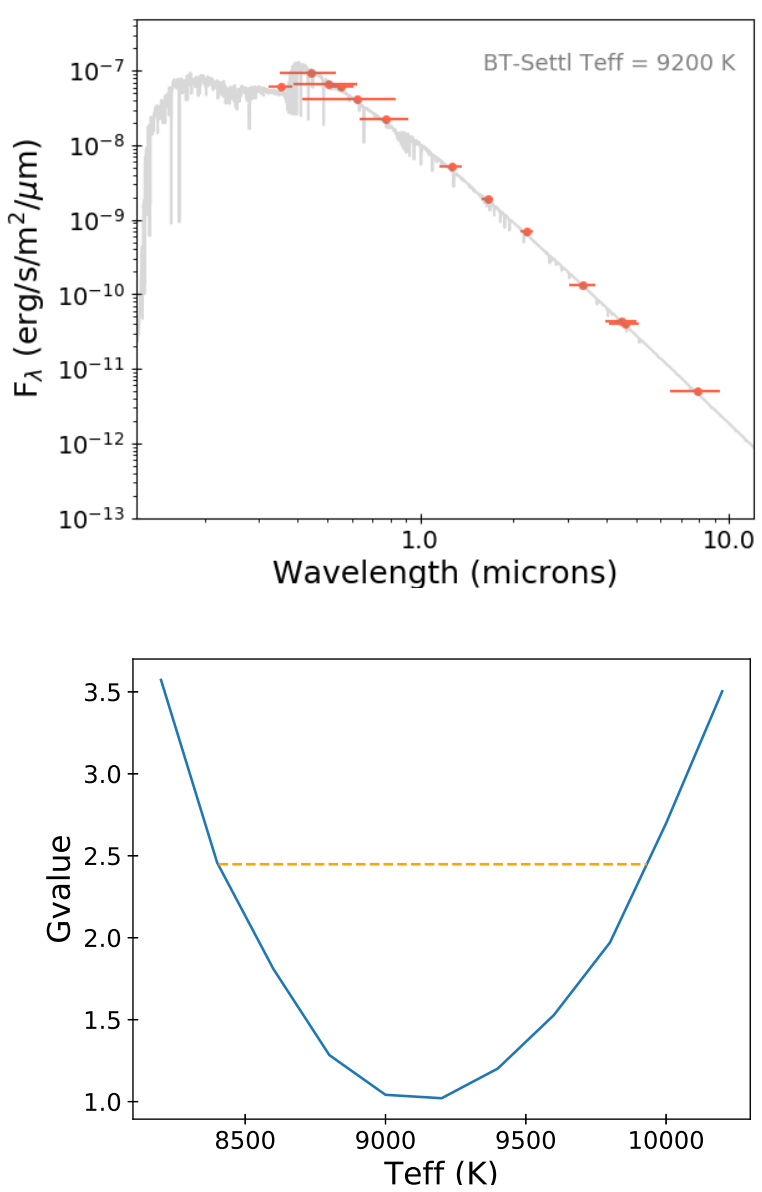

Fig. 7. Spectral energy distribution (SED) of the primary HIP 79124 A0V star. Top panel: a BT-Settl model (Allard et al. 2012) of $T_{\text {eff }}=9200 \mathrm{~K}$ and $\log g=4.5 \mathrm{dex}$ is fitted to the flux values at $\leq 10 \mu \mathrm{m}$ compiled from the literature (see Table 4). Bottom panel: G-value (Cushing et al. 2008) for BT-Settl models with different $T_{\text {eff }}$. The orange dashed line shows the upper limit for the considered well-fitting models.

The isochrones are sufficiently well-spaced to derive precise age estimates for each component. Early-type stars evolve onto the main sequence along a Henyey track both horizontally and vertically in an HR diagram. Close to the main-sequence "turnon", small errors in the temperature/luminosity can translate into larger uncertainties in the component age. Fortunately, as shown in Fig. 8, HIP 79124 A resides in a region on the HR diagram sufficiently away from the MS turn-on for early A stars: differences in predicted luminosities/temperatures for isochrones at 1$10 \mathrm{Myr}$ are significantly larger than measurement uncertainties. While uncertainties are larger for HIP 79124 BC, the vertical spacing for isochrones for low-mass stars is larger, $\gtrsim 0.1-0.2 \mathrm{dex}$.

Pecaut \& Mamajek (2016) reported an age spread of \pm 7 Myr for their adopted 10 Myr US subregion, finding an age gradient within the subgroup where stars are older as they blend with UCL. This seems to be consistent with a star-formation history that might explain the conflict in the derived ages between hot and cold stars (Fang et al. 2017). If this was indeed the case, the models would deliver the same age estimates for the three HIP 79124 objects. However, as presented in Table 5, we find that the primary seems to be consistently older for the models ( $\sim 6 \mathrm{Myr})$ than the B and $\mathrm{C}$ components ( $3 \mathrm{Myr})$. The PARSEC-COLIBRI isochrones differ significantly from the rest of models for the two low-mass companions, as it has also been the case in previous 

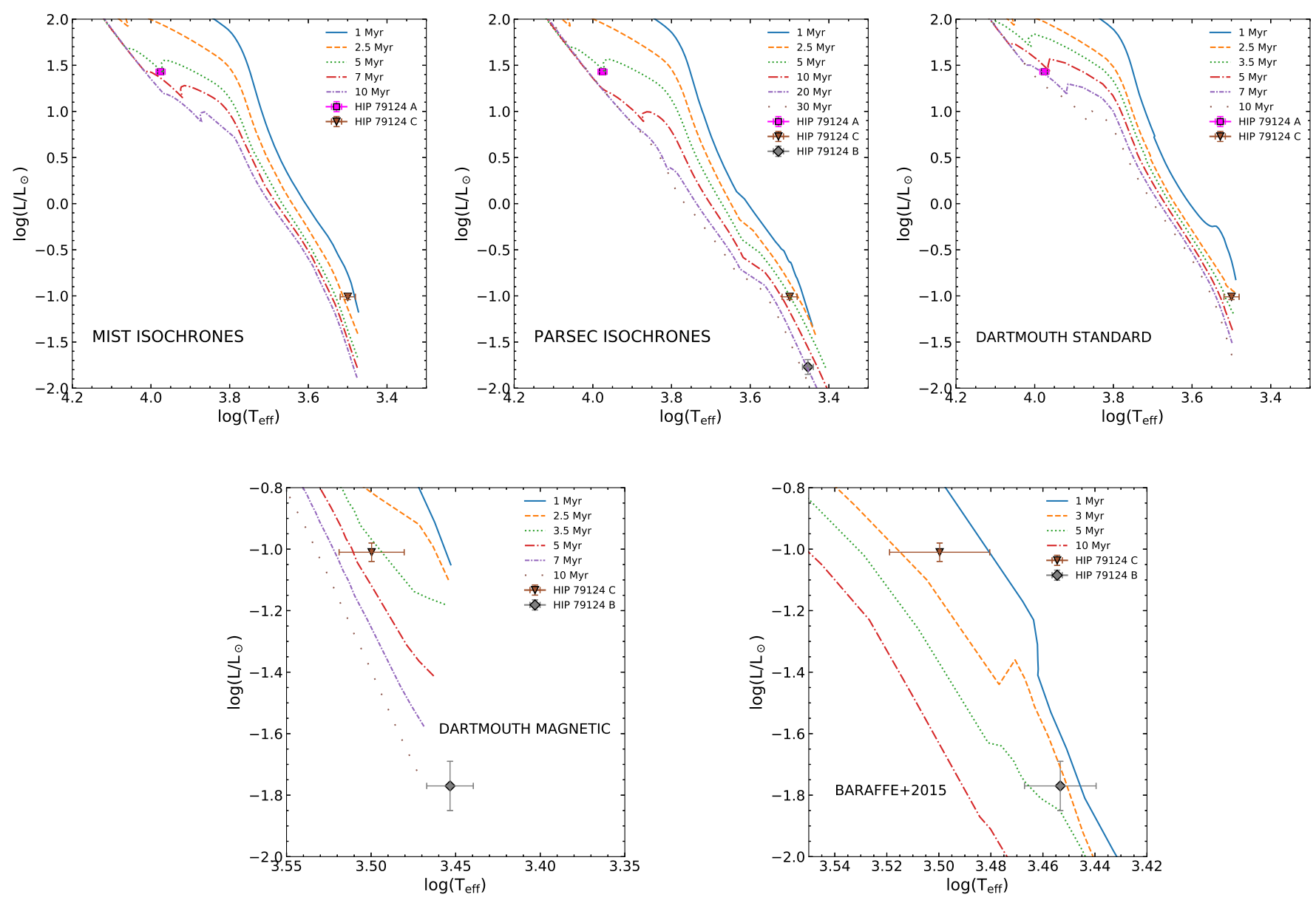

Fig. 8. Hertzsprung-Russell diagrams for the HIP 79124 triple system. The observed luminosity and temperature of the individual objects are tested against several theoretical models to derive an age estimate for the system. Numerical values are presented in Table 5.

studies (e.g., Kraus et al. 2015). This might be due to the artificial shift to the models made to fit the observed mass-radius relation for low-mass stars (Chen et al. 2014). For this reason, we do not consider their derived values to compute the mean age of HIP $79124 \mathrm{~B}$ and C.

The fact that the models predict a younger age for low-mass objects compared to the massive primary is in line with the results by Pecaut \& Mamajek (2016), where essentially all the objects with a later type than M0 are inferred to have an age below $5 \mathrm{Myr}$, moving to younger ages as the stars are cooler. Also, an age of $6 \mathrm{Myr}$ for the primary star is indeed expected from the location of the HIP 79124 system in the USco subregion. Figure 9 in Pecaut \& Mamajek (2016) shows a map of the spatial distribution of derived median-ages within the Sco-Cen complex. In this diagram, HIP 79124 falls exactly in the northern part of USco where stars tend to be younger than the mean age of the subregion.

The magnetic isochrones from Feiden (2016), which take into account the possible magnetic inhibition of convection in young low-mass stars, seem to provide a more compatible age estimate between the $\mathrm{A}$ and $\mathrm{C}$ components. Another effect linked to magnetic fields is the occurrence of spots on the surface of young stars. Spotted stars not only cause inflated radii at all ages, but they also experience a decrease in their luminosity and temperature (the latter especially for low-mass stars), which may produce a dispersion in the HR diagram. As found by Somers \& Pinsonneault (2015), this effect makes PMS stars appear younger and less massive when spots are present. Although the scatter does not seem to be high enough to explain the global age-mass discrepancy, it might be a contributor to take into account. In this way, we can apply the age correction factors derived in Somers \& Pinsonneault (2015) to our 3-Myr HIP 79124 B and C companions. Assuming that $1 / 6$ of the stellar surface is covered by spots, we find that $\mathrm{C}$ would have an age of $\sim 6 \mathrm{Myr}$ (for a corresponding mass of $\sim 250 M_{\text {Jup }}$, see Sect. 4.3). The B companion is not massive enough to derive a correction factor from the models, but its age would certainly lay beyond $6 \mathrm{Myr}$. This is very much in line with the observed age of the primary star. Indeed, the fact that models do not reproduce the effect of magnetic fields, which slow down the contraction of PMS stars and affect their luminosity and temperature, appears to explain our results well.

Another source of uncertainty in the PMS ages arises from the physical processes that contribute to the initial position of the star in the HR diagram at $t=0$, from which the contraction follows. The radius at which the contraction starts varies with early accretion rate, which creates a spread in radii (and luminosities) with which the stars of different masses are born (e.g., Hartmann 2003; Soderblom et al. 2014). This introduces uncertainties on the contraction ages, especially for intermediate-mass stars (Hartmann et al. 2016). In the case of HIP 79124 A, a shift in luminosity of $\log \left(L / L_{\odot}\right) \sim 0.5 \mathrm{dex}$ would be required for it to match the $\sim 3 \mathrm{Myr}$-old isochrone that better fits the age of the low-mass companions. This means that for the triple system to have common ages, the birthline for intermediate-mass 


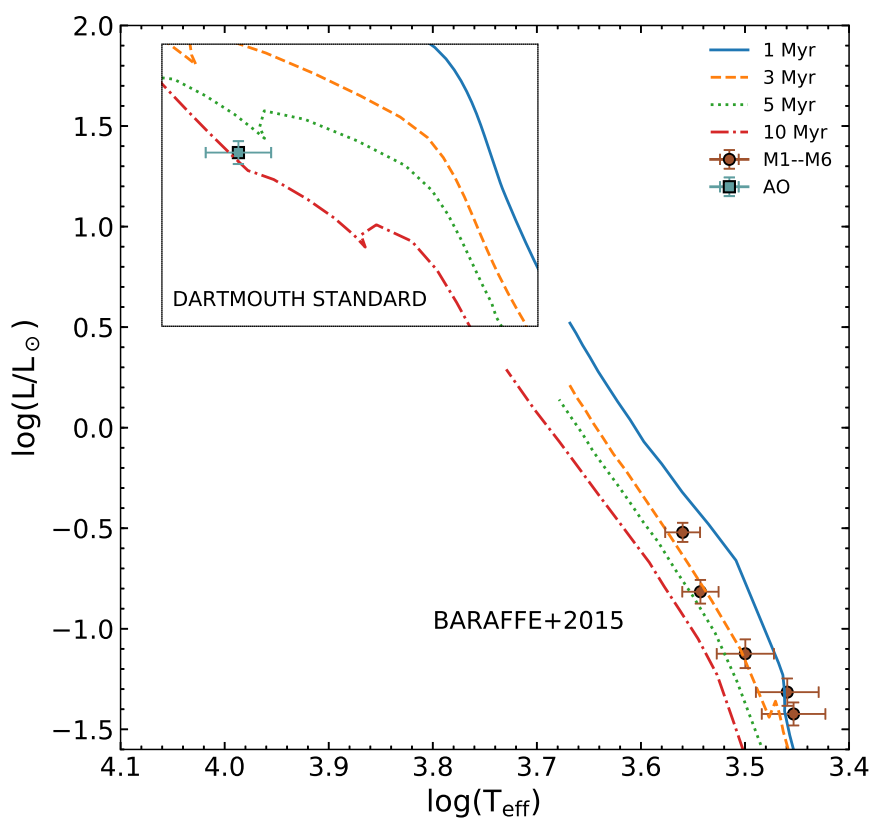

Fig. 9. Hertzsprung-Russell diagram for the five M-type stars found within $10^{\prime}$ from the location of HIP 79124 A. The BHAC15 isochrones (Baraffe et al. 2015) are used to estimate their age. We also include the closest A0-type star and confront it against the Dartmouth standard models (Dotter et al. 2008). The inner and the outer figures share axes.

stars would need to be corrected to a lower luminosity level by a factor of $\sim 3$.

Finally, in a similar fashion to what has been done for the HIP 79124 companions, we have obtained an estimation of the luminosity and temperature of the stars in the vicinity (within $10^{\prime}$ ) of HIP 79124 A. We found five M-type stars members of USco confirmed by the BANYAN $\Sigma$ tool (Gagne et al. 2018), which are confronted in Fig. 9 against the BHAC15 isochrones. When no extinction factors were available or we could not calculate them, we adopted the same extinction as for HIP 79124 $\mathrm{A}$, and an uncertainty of $0.5 \mathrm{mag}$. If the calculated extinction was non-physical, i.e., negative, $A_{V}$ was set to zero. We have also included the closest massive star to HIP 79124 A, which also happens to be an A0-type star at about $28^{\prime}$, compared to the Dartmouth standard models. In the same way as for the HIP 79124 system, the low-mass stars tend to give an age of $\sim 3 \mathrm{Myr}$, about half of the age that the Dartmouth models estimate for the A0 star (5-10 Myr).

\subsection{Mass of the HIP 79124 triple system}

We thus consider an age of $6 \pm 1$ Myr for HIP 79124, derived from the primary A0-type star. With this parameter well constrained, the observed $J H K_{\mathrm{s}} L_{\mathrm{p}}$ photometry presented in Tables 2 and 3 and the excellent accuracy in the distance to the system taken from Gaia-DR2, we can derive the mass of B and C using the BHAC15 isochrones (Baraffe et al. 2015).

Figure 10 shows an approximate mass for the low-mass companions using $J H K_{\text {s }}$ photometry derived from our CHARIS data and Keck/NIRC2 L'photometry. The close M6-type B companion agrees well with a mass of $\sim 100 M_{\text {Jup }}$. Hinkley et al. (2015) reported a mass of $\sim 135 M_{\text {Jup }}$ for an age of $10 \mathrm{Myr}$ from $\mathrm{L}_{\mathrm{p}}$ observations, as we similarly obtain for $6 \mathrm{Myr}$. It is interesting to note that this object seems to be slightly brighter in $J$ and $\mathrm{L}_{\mathrm{p}}$ bands than in $H$ and $K_{\mathrm{s}}$. The effect in $J$ can be explained by the object simply being bright in this band, or it might also be due to slight contamination from the primary star (see Sect. 4.1.2), while in $L_{\mathrm{p}}$ the object appears to be somewhat red compared to what it is predicted by BT-Settl models (Allard et al. 2012; Baraffe et al. 2015). C is more massive and it is found to fall at $\sim 350 M_{\text {Jup }}$ in all bands, which proves the consistency of our derived $J H K_{\mathrm{s}}$ photometry coupled with the extracted $L_{p}$ magnitudes from Keck/NIRC2 archival data. If we considered an age of the system of $3 \mathrm{Myr}$, as obtained from the models for the low-mass companions, $\mathrm{B}$ and $\mathrm{C}$ would have respective estimated masses of $\sim 55 M_{\text {Jup }}$ and $\sim 250 M_{\text {Jup }}$. Applying the correction for spotted stars from Somers \& Pinsonneault (2015) for the C companion, its mass would be of the order of $\sim 310 M_{\text {Jup }}$, very close to the mass obtained using the age derived from the primary, which indicates that magnetic fields have an important role in the observed discrepancy.

This same effect can be translated to the inferred masses of exoplanets discovered by direct imaging in star-forming regions. For instance, the recent planetary-mass companion revealed within the transition disk around the $\sim 5$ Myr-old PDS 70 star (Keppler et al. 2018) is estimated to have a mass of $\sim 5 M_{\text {Jup }}$ from its photometry, using the hot-start COND models (Baraffe et al. 2003). As PDS 70 is a low-mass K7-type star in Upper Centaurus-Lupus group (with a mean age of $16 \pm 2 \mathrm{Myr}$; Pecaut \& Mamajek 2016), one might hypothesize that the real age of the system could be underestimated. If PDS 70 was older by a factor of 2, as we see for HIP 79124, the planetary-mass companion would be more massive, of $\sim 7 M_{\text {Jup }}$, using again the COND models. Similarly, the ROXs 42B T Tauri binary star with an M0-type primary, is a member of the $\rho$ Oph complex, and hosts a directly imaged companion. According to the COND models, this circumbinary object lies in the planetarymass regime with $\sim 10 M_{\text {Jup }}$ (Currie et al. 2014b). If instead of $\sim 2.5$ Myr the system age was twice of that, the mass of the planet would increase up to $\sim 13 M_{\text {Jup }}$.

\subsection{Formation scenario}

Given the stellar nature of the companions, the natural approach to study the formation path of the low-mass stars would be to consider the fragmentation of the molecular cloud that gave origin to the HIP 79124 system. Indeed, radiation hydrodynamical calculations by Bate (2012) show that the initial mass function (IMF) of the formed objects peak at about the masses of HIP $79124 \mathrm{~B}$ and C. These simulations also reproduce a formation timescale for a massive A-type star that is very similar (well below 1 Myr difference) to that of low-mass objects down to the brown dwarf regime. Triple systems however seem to be rare, with a frequency of $\sim 4 \%$, as also found by observational results (e.g., Daemgen et al. 2015), and a separation distribution culminating at $\sim 100 \mathrm{AU}$.

An intriguing possibility is that the two low-mass stellar companions are formed via gravitational instability (GI). In this scenario, a massive and cold disk may gravitationally collapse and break down in fragments of sizes ranging from planetarymass companions to low-mass stars on wide orbits (e.g., Boss 1998; Rafikov \& Goldreich 2005). For one of these fragments to form, the cooling timescale needs to be shorter than the orbital period, which also assures coevality of the triple system in the GI scenario (Gammie 2003). Recent models are able to generate a synthetic population of GI-formed objects, and dynamically evolve the system before and after disk dispersal (Forgan \& Rice 2013; Forgan et al. 2015, 2018). These predictions have been tested against high-contrast direct imaging data, showing that, if 

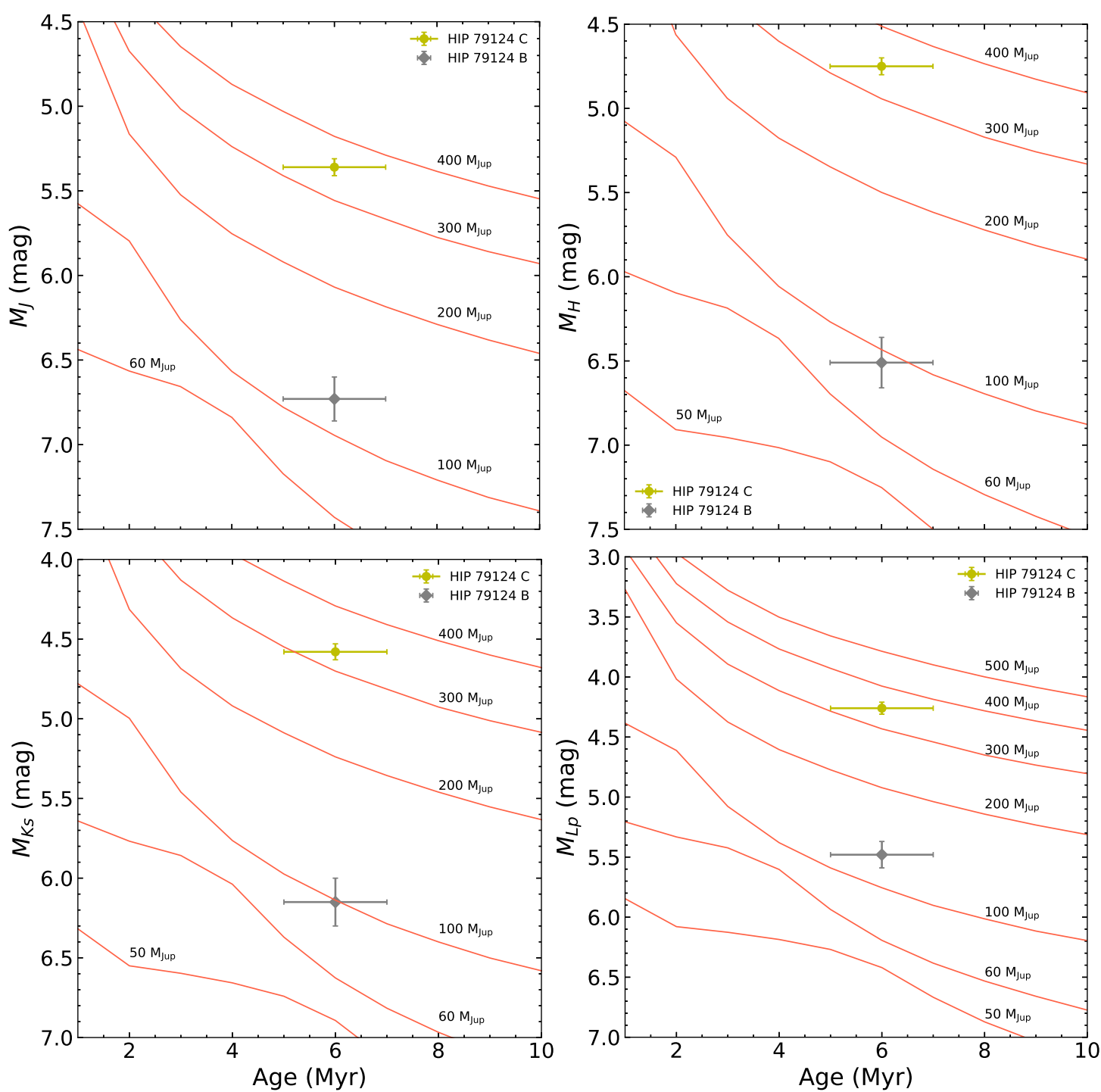

Fig. 10. Absolute magnitude vs Age of the HIP 79124 low-mass comapnions for $J H K_{\mathrm{s}} L^{\prime}$. Baraffe et al. (2015) models are overplotted to estimate their masses. The age of the companions is set at $6 \pm 1 \mathrm{Myr}$, taken from the primary age estimation (see Fig. 8 and Table 5).

substellar objects at separations $>30 \mathrm{AU}$ are indeed formed via GI, this formation method is rare (Vigan et al. 2017).

For solar-mass stars with protoplanetary disks extending up to $100 \mathrm{AU}$, models by Forgan et al. (2018) resulted in the formation of companions as massive as $\sim 120 \mathrm{M}_{\mathrm{Jup}}$. Stamatellos \& Whitworth (2009) also showed that the fragmentation of a $400 \mathrm{AU}$ disk around a $0.7 M_{\odot}$ star can give rise to a broad range of companions, $30 \%$ of them being low-mass stars (up to $\sim 200 M_{\text {Jup }}$ ). This indeed could be the formation process to explain the location of HIP $79124 \mathrm{~B}, \mathrm{a} \sim 100 M_{\text {Jup }}$ star located at a projected separation of only $\sim 25 \mathrm{AU}$.

\section{Conclusions}

We have presented the first spectrophotometric study of the USco HIP 79124 triple system with SCExAO/CHARIS. Combining low-resolution $J H K_{\mathrm{s}}$ spectroscopy with archival $L^{\prime}$ photometry from Hinkley et al. (2015) and Serabyn et al. (2017), we estimate the spectral types of the companions, which altogether serves as a diagnostic to derive the age of the system and the masses of the low-mass objects. The key results of our analysis can be summarized as
Table 5. Model age estimates of the triple system (in Myr).

\begin{tabular}{lccc}
\hline \hline Model & A & B & C \\
\hline MIST & $5 \pm 1$ & - & $2 \pm 1$ \\
PARSEC-COLIBRI & $6 \pm 1$ & $17 \pm 5$ & $5_{-2.5}^{+5}$ \\
Dartmouth std & $6 \pm 1$ & - & $3 \pm 1$ \\
Dartmouth mag & - & - & $4_{-1}^{+3}$ \\
Baraffe+2015 & - & $3 \pm 2$ & $2 \pm 1$ \\
\hline Mean & $6 \pm 1$ & $3 \pm 2$ & $3 \pm 1$ \\
\hline
\end{tabular}

Notes. The PARSEC-COLIBRI isochrones are not considered in the computation of the mean age of the $\mathrm{B}$ and $\mathrm{C}$ low-mass companions (see text).

- SCExAO/CHARIS detects HIP 79124 B and C in lowresolution mode without the employment of any PSFsubtraction algorithm at a S/N of $\sim 9$ and $\sim 120$ and at distances of $\sim 0.18^{\prime \prime}$ and $\sim 0.97^{\prime \prime}$, respectively.

- We account for the correlated noise present in IFS data (Greco \& Brandt 2016). B falls in a highly-correlated regime, even for well-separated wavelength channels. In the 
Table 6. Parameters of the HIP 79124 triple system.

\begin{tabular}{ccccccccc}
\hline \hline HIP 79124 & $\begin{array}{c}\text { Distance } \\
(\mathrm{pc})\end{array}$ & Spectral type & $\begin{array}{c}T_{\text {eff }} \\
(\mathrm{K})\end{array}$ & $\begin{array}{c}\log \left(L / L_{\odot}\right) \\
(\mathrm{dex})\end{array}$ & $\begin{array}{c}\text { Age } \\
(\mathrm{Myr})\end{array}$ & $\begin{array}{c}\text { Mass } \\
\left(M_{\text {Jup }}\right)\end{array}$ & $\begin{array}{c}\text { Sep. } \\
(\mathrm{mas})\end{array}$ & $\begin{array}{c}\text { PA } \\
(\mathrm{deg})\end{array}$ \\
\hline $\mathrm{A}$ & $137.0 \pm 1.2^{a}$ & $\mathrm{~A} 0 \mathrm{~V}^{b}$ & $9450 \pm 250$ & $1.43 \pm 0.03$ & $6 \pm 1$ & - & - & - \\
$\mathrm{B}$ & - & $\mathrm{M} 6 \pm 0.5$ & $2840 \pm 90$ & $-1.77 \pm 0.08$ & $3 \pm 2$ & $100 \pm 30$ & $180 \pm 5$ & $252.9 \pm 1.6$ \\
$\mathrm{C}$ & - & $\mathrm{M} 4 \pm 0.5$ & $3160 \pm 140$ & $-1.01 \pm 0.03$ & $3 \pm 1$ & $330 \pm 30$ & $967 \pm 6$ & $100.39 \pm 0.03$ \\
\hline
\end{tabular}

Notes. ${ }^{(a)}$ From the Gaia-DR2 (Lindegren et al. 2018). ${ }^{(b)}$ From Houk \& Smith-Moore (1988).

case of the outer $\mathrm{C}$ companion, the uncorrelated amplitude is predominant. Using these correlated errors, we find that young ( $10 \mathrm{Myr})$ standard objects from Luhman et al. (2017) best match the spectra of B (M6) and C (M4).

- We assemble an HR diagram where we place the triple system, and confront their luminosity- $T_{\text {eff }}$ values with several theoretical models to assess a common age estimate. However, the primary star is found to have an age of $\sim 6 \mathrm{Myr}$, while the models consistently deliver about half this age for the low-mass companions.

- This age-mass discrepancy for young low-mass stars is in line with the results seen in several young regions such as Sco-Cen (Pecaut \& Mamajek 2016). As HIP 79124 should be coeval with the three objects forming in timescales $<1 \mathrm{Myr}$ (Bate 2012), this result strongly points towards the fact that the models do not reproduce well enough the PMS phase of low-mass stars.

- Adopting the age of the primary star for the entire system, we find a mass of $\mathrm{B}$ of $\sim 100 M_{\text {Jup }}$, and $\sim 330 M_{\text {Jup }}$ for $\mathrm{C}$. Given their masses and small orbital separation, there is the possibility that these objects formed via disk instability (e.g., Forgan et al. 2018).

- This effect can alter the mass of the directly-imaged companions to low-mass stars, if the age of the system is derived from isochronal fits to the photometric data of the host star.

We have demonstrated the SCExAO/CHARIS capabilities by resolving a very packed system and constraining their properties through low-resolution spectroscopy. For this very likely coeval system, models predict an older age for the A0-type primary star than for the low-mass companions. This result might be related to magnetic field effects, which implies that the models do not reproduce with enough accuracy the contraction rate of low-mass PMS stars or the presence of stellar spots, and thus deliver a younger age (Somers \& Pinsonneault 2015; Feiden 2016; Somers \& Stassun 2017). Further observations of this system could constrain the orbit of the $\mathrm{B}$ companion to derive its period and a more reliable mass estimate.

Acknowledgements. We thank Kevin Luhman, Eric Mamajek, and Mark Pecaut for helpful draft comments. We wish to emphasize the pivotal cultural role and reverence that the summit of Maunakea has always had within the indigenous Hawaiian community. We are most fortunate to have the privilege to conduct scientific observations from this mountain. R.A.-T. and M.J. gratefully acknowledge funding from the Knut and Alice Wallenberg foundation. S.D. acknowledges support from the "Progetti Premiali" funding scheme of the Italian Ministry of Education, University, and Research. E. A. is supported by MEXT/JSPS KAKENHI grant No. 17K05399. M.T. is supported by MEXT/JSPS KAKENHI grant Nos. 18H05442, 15H02063, and 22000005.M. $\mathrm{H}$. is supported by the Grant-in-Aid for Scientific Research on Innovative Areas (2302, 23103002) under the Ministry of Education, Culture, Sports, Science and Technology (MEXT) of Japan. This research has benefited from the Montreal Brown Dwarf and Exoplanet Spectral Library, maintained by Jonathan Gagne. This work has made use of data from the European Space Agency (ESA) mission Gaia (https://www.cosmos.esa.int/gaia), processed by the Gaia Data Processing and Analysis Consortium (DPAC, https://www.cosmos.esa. int/web/gaia/dpac/consortium). Funding for the DPAC has been provided by national institutions, in particular the institutions participating in the Gaia Multilateral Agreement.

\section{References}

Allard, F., Homeier, D., \& Freytag, B. 2012, Phil. Trans. Roy. Soc. A, 370, 2765 Allers, K. N., \& Liu, M. C. 2013, ApJ, 772, 79

Barman, T. S., Macintosh, B., Konopacky, Q., \& Marois, C. 2011, ApJ, 735, L39 Bell, C. P. M., Mamajek, E. E., \& Naylor, T. 2015, MNRAS, 454, 593 Baraffe, I., Chabrier, G., Barman, T. S., et al. 2003, A\&A, 402, 701 Baraffe, I., Homeier, D., Allard, F., et al. 2015, A\&A, 577, A42 Bate, M. R. 2012, MNRAS, 419, 3115

Biller, B., \& Bonnefoy, M. 2018, in Handbook of Exoplanets, (Springer International Publishing AG), 101

Bonnefoy, M., Chauvin, G., Lagrange, A. M., et al. 2014, A\&A, 562, A127 Boss, A. P. 1998, ApJ, 503, 923

Bowler, B. 2016, PASP, 128, 2001

Brandt, T. D., Rizzo, M., Groff, T., et al. 2017, J. Astron. Telesc. Instrum. Syst., 3, 048002

Caffau, E., Ludwig, H.-G., Steffen, M., et al. 2011, Sol. Phys., 268, 255 Canty, J. I., Lucas, P. W., Roche, P. F., et al. 2013, MNRAS, 435, 2650 Carpenter, J. M. 2001, AJ, 121, 2851

Carpenter, J. M., Mamajek, E. E., Hillenbrand, L. A., et al. 2006, ApJ, 651, 49 Chen, Y., Girardi, L., Bressan, A., et al. 2014, MNRAS, 444, 2525

Choi, J., Dotter, A., Conroy, C., et al. 2016, ApJ, 823, 102

Cook, N. J., Scholz, A., \& Jayawardhana, R. 2017, AJ, 154, 256 Currie, T., Burrows, A., Itoh, Y., et al. 2011, ApJ, 729, 128

Currie, T., Daemgen, S., Debes, J. H., et al. 2014a, ApJ, 780, L30

Currie, T., Burrows, A., \& Daemgen, S. 2014b, ApJ, 787, 104

Currie, T., Cloutier, R., Brittain, S., et al. 2015, ApJ, 814, L27

Currie, T., Brandt, T. D., Uyama, T., et al. 2018a, AJ, 156, 291

Currie, T., Kasdin, N. J., Groff, T. D., et al. 2018b, PASP, 130, 4505

Cushing, M. C., Marley, M. S., Saumon, D., et al. 2008, ApJ, 678, 1372

Cutri, R. M., Skrutskie, M. F., van Dyk, S., et al. 2003, VizieR Online Data Catalog: II/246

Cutri, R. M., Wright, E. L., Conrow, T., et al. 2012, VizieR Online Data Catalog: II/311

Daemgen, S., Bonavita, M., Jayawardhana, R., et al. 2015, ApJ, 799, 155 de Zeeuw, P. T., Hoogerwerf, R., de Bruijne, J. H. J., et al. 1999, AJ, 117, 354 Donaldson, J. K., Weinberger, A. J., Gagne, J., et al. 2016, ApJ, 833, 95 Dotter, A. 2016, ApJS, 222, 8

Dotter, A., Chaboyer, B., Jevremovic, D., et al. 2008, ApJS, 178, 89 Eikenberry, S., Elston, R., Raines, S., et al. 2004, SPIE, 5492, 1196 Faherty, J. K., Riedel, A. R., Cruz, K. L., et al. 2016, ApJS, 225, 10 Fang, Q., Herczeg, G. J., \& Rizzuto, A. 2017, ApJ, 842, 123 Feiden, G. A. 2016, A\&A, 593, A99

Fiorucci, M., \& Munari, U. 2003, A\&A, 401, 781

Forgan, D., \& Rice, K. 2013, MNRAS, 432, 3168

Forgan, D., Parker, R. J., \& Rice, K. 2015, MNRAS, 447, 836

Forgan, D. H., Hall, C., Meru, F., et al. 2018, MNRAS, 474, 5036

Gagne, J., Faherty, J. K., Cruz, K. L., et al. 2015, ApJS, 219, 33

Gagne, J., Mamajek, E. E., Malo, L., et al. 2018, ApJ, 856, 16

Gaia Collaboration (Brown, A. G. A., et al.) 2018, A\&A, 616, A1

Gammie, C. F. 2003, ApJ, 553, 174

Garcia, E. V., Currie, T., Guyon, O., et al. 2017, ApJ, 834, 162

Golimowski, D. A., Leggett, S. K., Marley, M. S., et al. 2004, AJ, 127, 3516

Greco, J. P., \& Brandt, T. D. 2016, ApJ, 833, 134

Groff, T., Peters, M., \& Kasdin, N. 2013, Am. Astron. Soc., 221, 345.10

Hartmann, L. 2003, ApJ, 585, 398

Hartmann, L., Herczeg, G., \& Calvet, N. 2016, ARA\&A, 54, 135

Herczeg, G. J., \& Hillenbrand, L. A. 2015, ApJ, 808, 23

Hillenbrand, L. A. 1997, AJ, 113, 1733

Hinkley, S., Kraus, A. L., Ireland, M. J., et al. 2015, ApJ, 806, 9 
Houk, N., \& Smith-Moore, M. 1988, Michigan Spectral Survey (Ann Arbor, MI Dept. of Astronomy, Univ. of Michigan), 4

Jordi, C., Gebran, M., Carrasco, J. M., et al. 2010, A\&A, 523, A48

Jovanovic, N., Martinache, F., Guyon, O., et al. 2015, PASP, 127, 890

Keppler, M., Benisty, M., Müller, A., et al. 2018, A\&A, 617, A44

Kouwenhoven, M. B. N., Brown, A. G. A., Zinnecker, H., et al. 2005, A\&A, 430, 137

Kouwenhoven, M. B. N., Brown, A. G. A., \& Kaper, L. 2007, A\&A, 464, 581

Kraus, A. L., \& Hillenbrand, L. A. 2007, AJ, 134, 2340

Kraus, A. L., \& Hillenbrand, L. A. 2009, ApJ, 704, 531

Kraus, A. L., Cody, A. M., Covey, K. R., et al. 2015, ApJ, 807, 3

Lafreniere, D., Jayawardhana, R., van Kerkwijk, M. H., et al. 2014, ApJ, 785, 47

Lindegren, L., Hernandez, J., Bombrun, A., et al. 2018, A\&A, 616, A2

Luhman, K. L., \& Mamajek, E. E. 2012, ApJ, 758, 31

Luhman, K. L., Stauffer, J. R., Muench, A. A., et al. 2003, ApJ, 593, 1093

Luhman, K. L., Mamajek, E. E., Shukla, S. J., et al. 2017, AJ, 153, 46

Luhman, K. L., Herrmann, K. A., Mamajek, E. E., et al. 2018, ApJ, 156, 76

Mamajek, E. E. 2005, ApJ, 634, 1385

Mann, A. W., \& von Braun, K. 2015, PASP, 127, 102

Marigo, P., Girardi, L., Bressan, A., et al. 2017, ApJ, 835, 77

Marois, C., Lafreniere, D., \& Doyon, R. 2006, ApJ, 641, 556

Mawet, D., Milli, J., Wahhaj, Z., et al. 2014, ApJ, 792, 97

McLean, I. S., McGovern, M. R., Burgasser, A. J., et al. 2003, ApJ, 596, 561

Myers, J. R., Sande, C. B., Miller, A. C., et al. 2015, Vizier Online Data Catalog: $\mathrm{V} / 145$

Paxton, B., Bildsten, L., Dotter, A., et al. 2011, ApJS, 192, 3

Paxton, B., Cantiello, M., Arras, P., et al. 2013, ApJS, 208, 4

Paxton, B., Marchant, P., Schwab, J., et al. 2015, ApJS, 220, 15

Pecaut, M. J., \& Mamajek, E. E. 2013, ApJS, 208, 9

Pecaut, M. J., \& Mamajek, E. E. 2016, MNRAS, 461, 794

Pecaut, M. J., Mamajek, E. E., Bubar, E. J., et al. 2012, ApJ , 746, 154

Perrin, M., Maire, J., Ingraham, P., et al. 2014, SPIE, 9147, 3

Peters, M., Groff, T., Kasdin, N. J., et al. 2012, SPIE, 8446, 7

Pickles, A. J. 1998, PASP, 110, 863

Preibisch, T., \& Mamajek, E. 2008, Handbook of Star Forming Regions, Volume

II, The Nearest OB Association: Scorpius-Centaurus (Sco OB2)

Preibisch, T., Brown, A., Bridges, T., et al. 2002, AJ, 124, 404

Quijada, M. A., Marx, C. T., Arendt, R. G., et al. 2004, SPIE, 5487, 244

Rafikov, R. R., \& Goldreich, P. 2005, ApJ, 631, 488

Rayner, J. T., Toomey, D. W., Onaka, P. M., et al. 2003, PASP, 115, 362

Rayner, J. T., Cushing, M. C., Vacca, W. D., et al. 2009, ApJS, 185, 289

Robert, J., Gagne, J., Artigau, E., et al. 2016, ApJ, 830, 144

Rodriguez, D. R., Zuckerman, B., Kastner, J. H., et al. 2013, ApJ, 774, 101

Serabyn, E., Huby, E., Matthews, K., et al. 2017, ApJ, 153, 43

Siess, L., Dufour, E., \& Forestini, M. 2000, A\&A, 358, 593

Soderblom, D. R., Hillenbrand, L. A., Jeffries, R. D., et al. 2014, in Protostars and Planets VI, eds. H. Beuther, R. S. Klessen, C. P. Dullemond, \& T. Henning (Tucson: University of Arizona Press), 219

Somers, G., \& Pinsonneault, M. H. 2015, ApJ, 807, 174

Somers, G., \& Stassun, K. G. 2017, AJ, 153, 101

Stamatellos, D., \& Whitworth, A. P. 2009, MNRAS, 392, 413

Thalmann, C., Carson, J., Janson, M., et al. 2009, ApJ, 707, 123

Thalmann, C., Janson, M., Buenzli, E., et al. 2013, ApJ, 763, 29

Thalmann, C., Desidera, S., Bonavita, M., et al. 2014, A\&A, 572, A91

Vigan, A., Bonavita, M., Biller, B., et al. 2017, A\&A, 603, A3

Webb, R. A., Zuckerman, B., Platais, I., et al. 1999, ApJ, 512, 63
1 Department of Astronomy, Stockholm University, AlbaNova University Center, 10691 Stockholm, Sweden e-mail: ruben.torres@astro.su.se

2 NASA-Ames Research Center, Moffett Field, California 94035, USA

3 National Astronomical Observatory of Japan, Subaru Telescope, National Institutes of Natural Sciences, Hilo, HI 96720, USA

${ }^{4}$ Eureka Scientific, 2452 Delmer Street Suite 100. Oakland, CA 94602-3017, USA

5 INAF-Osservatorio Astronomico di Padova, Vicolo dell'Osservatorio 5, 35122 Padova, Italy

${ }^{6}$ Astrobiology Center, National Institutes of Natural Sciences, 2-21-1 Osawa Mitaka, Tokyo, Japan

7 National Astronomical Observatory of Japan, 2-21-1, Osawa, Mitaka, Tokyo 181-8588, Japan

8 Institute for Astronomy, University of Hawaii, Hilo, HI, USA

9 Department of Physics, University of California-Santa Barbara, Santa Barbara, CA, USA

10 Steward Observatory, University of Arizona, Tucson, AZ 85721, USA

11 College of Optical Sciences, University of Arizona, Tucson, AZ 85721, USA

12 NASA-Goddard Space Flight Center, Greenbelt, MD, USA

13 Department of Mechanical Engineering, Princeton University, Princeton, NJ, USA

14 Department of Physics, University of Notre Dame, 225 Nieuwland Science Hall Notre Dame, IN 46556, USA

15 Department of Astronomy, California Institute of Technology, 1200 E. California Blvd., Pasadena, CA 91125, USA

16 Université Côte d'Azur, Observatoire de la Côte d'Azur, CNRS, Laboratoire Lagrange, France

17 Center for Extrasolar Planetary Systems, Space Science Institute, 1120 Paxton Ave., Cincinnati, OH 45208, USA

18 Jet Propulsion Laboratory, California Institute of Technology, 4800 Oak Grove Drive, Pasadena, CA 91109, USA

19 Institute for the Advancement of Higher Education, Hokkaido University, Kita 17, Nishi 8, Kita-ku, Sapporo 060-0817, Japan

20 ISAS/JAXA, 3-1-1 Yoshinodai, Chuo-ku, Sagamihara, Kanagawa 252-5210, Japan

21 Department of Astronomy, Graduate School of Science, The University of Tokyo, 7-3-1, Hongo, Bunkyo-ku, Tokyo 113-0033, Japan

22 Department of Astronomy, The Graduate University for Advanced Studies, National Astronomical Observatory of Japan, Japan

23 Max Planck Institut für Astronomie, Königstuhl 17, 69117 Heidelberg, Germany

24 Department of Astronomy, Graduate School of Science, The University of Tokyo, 7-3-1, Hongo, Bunkyo-ku, Tokyo 113-0033, Japan 